\title{
NRPs and circumstellar activity in HD 127972^
}

\author{
R. S. Levenhagen ${ }^{1}$, N. V. Leister ${ }^{1}$, J. Zorec ${ }^{2}$, E. Janot-Pacheco ${ }^{1}$, A. M. Hubert ${ }^{3}$, and M. Floquet ${ }^{3}$ \\ ${ }^{1}$ Instituto de Astronomia, Geofísica e Ciências Atmosféricas da Universidade de São Paulo, CUASO 05508-900 São Paulo, \\ Brazil \\ 2 Institut d'Astrophysique de Paris, 98bis Boulevard Arago, 75014 Paris, France \\ 3 Observatoire de Paris-Meudon, GEPI, FRE/2459, 92195 Meudon Cedex, France
}

Received 8 July 2002 / Accepted 19 December 2002

\begin{abstract}
Line profile variations (lpv) in $\mathrm{He}_{\mathrm{I}}, \mathrm{Fe}_{\text {II }}, \mathrm{Mg}$ II and Si II transitions were detected in the Be star $\eta$ Cen (HD 127972) by means of high resolution and $S / N$ spectroscopic observations obtained in six epochs from May 1996 to April 2001. They were interpreted in terms of nonradial pulsations (NRPs). Time analysis was performed using Cleanest algorithm and showed the following frequencies with high order of significance: $0.61 \mathrm{c} / \mathrm{d}, 1.48 \mathrm{c} / \mathrm{d}, 3.81 \mathrm{c} / \mathrm{d}, 5.31 \mathrm{c} / \mathrm{d}, 9.24 \mathrm{c} / \mathrm{d}$ and $10.35 \mathrm{c} / \mathrm{d}$. From phase variation diagrams we estimated mode degrees $\ell$ in the range $3-8$. If the $10.35 \mathrm{c} / \mathrm{d}$ frequency is considered the first harmonic of $5.31 \mathrm{c} / \mathrm{d}$, the corresponding azimuthal number of the mode is $|m|=4 \pm 2$. Except for $0.61 \mathrm{c} / \mathrm{d}$, all other frequencies are compatible with NRPs. During the period of progressive activity enhancement in the He I 6678 line, a strengthening of Balmer emission lines was observed. From Mar. 12 to Mar. 23, 2000, we noticed rapid variations of both $\mathrm{H} \alpha$ peak separation and $V / R$ ratios. Using a simple model for the $\mathrm{H} \alpha$ line emission formation, we outlined an explanation for the season-averaged $\mathrm{H} \alpha$ emission variation in terms of changes of the mass density in the circumstellar envelope. The fundamental parameters of $\eta$ Cen were analyzed using several methods. The adopted ones account for the stellar fast rotation, which helped us not only to estimate the stellar rotational frequency, but also to show that the star is in the middle of its main sequence life span.
\end{abstract}

Key words. stars: emission-line, Be - stars: oscillations - stars: individual: $\eta$ Cen - stars: fundamental parameters techniques: spectroscopic - line: profiles

\section{Introduction}

The classic Be phenomenon has been known since the discovery of emission lines in the spectrum of $\gamma$ Cas by Secchi (1867). Although for a long time this phenomenon was considered to be limited to the B spectral type, today its characteristics are recognized to be present in other spectral types and so it could be named the "OBAe phenomenon", as it is shared by non-supergiant late $\mathrm{O}$ to early A spectral type stars that have shown at least once some emission in $\mathrm{H}$ Balmer lines (Frost \& Conti 1976; Andrillat et al. 1986; Marlborough 2000). Apart from the many spectroscopic, photometric, spectrophotometric and polarimetric characteristics seen in the spectral domain ranging from visible to the IR, which are attributed to both photospheric activity and circumstellar envelope (CE), Be stars stand out by their high rotation, though undercritical with $\Omega / \Omega_{\mathrm{c}} \sim 0.8$ (Chauville et al. 2001). For simplicity, we will refer in this paper to all of these stars and phenomena as "Be stars" and "Be phenomenon" respectively. Two main questions concern these objects: what is their nature and

\footnotetext{
Send offprint requests to: R. S. Levenhagen,

e-mail: savarino@astro.iag.usp.br

* Based on observations made at the MCT/LNA Observatory, Brasópolis - Brazil, and at ESO/La Silla - Chile.
}

evolutionary status in order to generate these characteristics, and how do they produce the CE? In the spatial UV spectrum of Be stars there are signatures indicating the presence of winds with average mass loss of the order of $10^{-9}$ to $10^{-11} M_{\odot} \mathrm{yr}^{-1}$ (Snow 1987). So, neither once assumed critical rotation (Struve 1931) nor a stationary wind alone can account for the total mass flux in the $\mathrm{CE}$ where particle densities are $\sim 10^{12} \mathrm{~cm}^{-3}$. Combined wind-rotation mechanisms were put forward to produce the CE (Lamers \& Pauldrach 1991; Bjorkman \& Cassinelli 1993). Contrasting with the above continuing mass loss related phenomena, evidence is accumulating on recurrent small-scale or moderate $\Delta M \lessgtr 10^{-11} M_{\odot}$ (cf. Hanuschik et al. 1993; Floquet et al. 2000, 2002) to large-scale $\Delta M \gtrsim 10^{-10} M_{\odot}$ discrete mass ejections (Hubert et al. 2000; Zorec et al. 2000a,b). Ando (1983, 1986), Kambe et al. (1993) and Rivinius et al. (1999) suggested that the coupling of two nonradial pulsations (NRP) modes could supply the energy required to produce discrete ejections. The detection of line profile variations (hereafter lpv) and their interpretation as NRP were made for the Be star $\eta$ Cen (HD 127972, B1.5Vne) for the first time by Janot-Pacheco et al. (1991) in the HeI $\lambda 6678 \AA$ line. Leister et al. (1994) detected at least 6 bumps moving across the same line and attributed it to a tesseral NRP mode with pulsational parameters $\ell=7$ and $|m|=6$, a phenomenon 
also discussed by Telting \& Schrijvers (1997b). Basing their studies of lpv of the He I $\lambda 6678 \AA$ line on $\eta$ Cen on time series analysis with "Clean" (Roberts et al. 1974) and "Cleanest" (Foster 1995) algorithms, Janot-Pacheco et al. (1999) showed that the star displayed a multiperiodic character.

Long-term (years) photometric variations of $\eta$ Cen were reported by Jaschek et al. (1964) and Feinstein \& Marraco (1979). Short time-scale photometric variations of this object were first reported by Cuypers et al. (1989). Though the latter were sometimes interpreted as due to corotating features, they can also be atributed to NRP due to effects of compression/expansion phenomena associated with the local temperature variations caused by the passage of waves through the stellar surface (Smith 1977).

The aim of this paper is to present new Fourier analysis of the lpv in He I $\lambda \lambda 4026,4121,4144,4388,4471,4922,6678 \AA$, Si II $\lambda 4131 \AA, \mathrm{Mg}_{\text {II }} \lambda 4481 \AA$ and $\mathrm{Fe}_{\text {II }} \lambda 5169 \AA$ Aines. On the other hand, this star also underwent photometric monitoring by the Hipparcos satellite from 1990 to 1992. The variations detected are worth studying in some detail. Observations of $\eta$ Cen were also made in the BCD spectrophotometric system, which allows us to derive an independent set of stellar fundamental parameters unperturbed by $\mathrm{CE}$ emission/absorption. Once these parameters are corrected for rotational effects, we can determine the evolutionary status of the central object. Their comparison with fundamental parameters derived using stellar model atmospheres will also help us to discuss the effects induced by the rapid rotation on the stellar surface. Finally, we will report $\mathrm{H} \alpha$ emission line profiles obtained from 1996 to 2001 whose variations can give us new insights on CE formation characteristics.

\section{Observations}

The high resolution and signal-to-noise spectra obtained are distributed over 30 nights. From May 1996 to May 2000 observations were performed during 17 nights with the Coudé spectrograph at the Brazilian Laboratório Nacional de Astrofísica (Pico dos Dias) (LNA) $1.60 \mathrm{~m}$ (B\&C) telescope using a EMI CCD camera $(1152 \times 770$ pixels $)$. For 13 nights, from April 2000 to April 2001, observations were carried out with the fiber-fed extended range optical spectrograph (FEROS) at the ESO - La Silla $1.52 \mathrm{~m}$ telescope. The LNA spectra were taken with a $1800 \mathrm{l} / \mathrm{mm}$ grating (first inverse order) centered on the He I $\lambda 6678 \AA$ line, with sporadic $\mathrm{H} \alpha(\lambda 6563 \AA)$ measurements. This arrangement yields a spectral resolution of $R \sim 40000$ with a reciprocal dispersion of $0.08 \AA /$ pixel. The signal-to-noise ratio is typically $\sim 250$ for exposure times around $300 \mathrm{~s}$. Bias, flat-field and $\mathrm{Th}-\mathrm{Ar}$ comparison lamp were taken during each night, and observations were reduced with the $\operatorname{IRAF}^{1}$ package. ESO spectra were taken in a spectral coverage of 3560-9200 $\AA$, recorded in 38 orders on the detector, with typical $S / N \sim 300$ (near He I $6678 \AA$ ). The resolution power of

\footnotetext{
${ }^{1}$ IRAF is distributed by the National Optical Astronomy Observatories, which is operated by the Association of Universities for Research in Astronomy (AURA), Inc., under cooperative agreement with the National Science Foundation.
}

FEROS is $R \sim 48000$. Spectra studied and shown in this paper are reduced to the heliocentric frame.

The $\log$ of spectroscopic observations carried out on HD 127972 is presented in Table 1. A selected sample of He I $6678 \AA$ line profiles obtained in each observing epoch is shown in Fig. 1. In the present paper we study only lpv associated with NRP. The temporary appearance of emissions in the outer line wings of the He I 6678 A line, dimples (Smith \& Polidan 1993), central quasi emissions, etc. will be discussed elsewhere.

\section{Analysis of observations}

\subsection{Algorithm used}

The search for lpv multiperiodicities in HD 127972 spectra was made by means of the Fast Fourier Transform (FFT) with the "Cleanest" algorithm (Foster 1995; Emilio 1997). It is known that the use of FFT without a frequency search criterion yields periodograms with a very large number of frequencies, where the signal appears convolved with the time sampling (aliasing), and the detection of periodicities becomes somewhat risky and uncertain. However, some constraints on the selection of the detected periods can be imposed by filtering signals from the data sampling with well-founded criteria. One of such methods is the Cleanest algorithm which considers the series as data vectors represented linearly on a given vectorial base. The method proceeds by steps by subtracting sequentially from the residuals, obtained once the FFT was applied to the vector data, a model function derived using each new frequency peak considered as statistically significant by a $\chi^{2}$ test. Once the last significant peak in a step cycle has been found, a new vectorial base is constructed with the last vectors plus the contribution of the last detected signal. The process goes on until there are no more statistically significant peaks or simply because the process reaches a stop condition, such as the imposed maximum number of possible frequencies. The last model function is formed by a basis with $2 n+1$ tentative functions. An important feature of Cleanest is that it does not assume the average signal present in each time residual to be zero, as does the "Clean" method (Roberts et al. 1987). The assumption of zeroaveraged signals in time residuals leads to the unprobable fact that the sample data are modulated by signals with an integer number of cycles which can hinder the performance of signal detection (Emilio 1997; Levenhagen 2000).

The reliability of the resulting power spectrum can be questioned by the time data sampling. It implies a finite extent of observational missions, an unequal time distribution of data and the strong 24 hours periodicity of data sampling. It is thus important to test the performance of the algorithm of time analysis used in adverse sampling conditions. This can be achieved in principle by applying the method to a set of synthetic spectra affected by the same noise and time distribution as the real spectra. In this way one can infer the effects of the convolution of the NRP signals with the spectral window. For this purpose, we created a set of 652 synthetic spectra composed of four sinusoidal signals, whose frequencies are the same as those detected in real data (see Sect. 3.2), namely $v=0.6$, 
Table 1. Log of HD 127972 spectroscopic observing campaigns.

\begin{tabular}{|c|c|c|c|c|c|c|}
\hline Epoch & $\begin{array}{l}\text { Observing } \\
\text { season }\end{array}$ & Telescope & Instrument & Spectral range & No of nights & No of spectra \\
\hline 1 & May/June 1996 & LNA $1.60 \mathrm{~m}$ & Coudé & He I $6678 \AA$ & 7 & 539 \\
\hline 2 & May 1997 & LNA $1.60 \mathrm{~m}$ & Coudé & He I $6678 \AA$ & 3 & 26 \\
\hline 3 & June 1998 & LNA $1.60 \mathrm{~m}$ & Coudé & He I $6678 \AA$ & 2 & 31 \\
\hline 4 & April 2000 & ESO $1.52 \mathrm{~m}$ & FEROS & $3560-9200 \AA$ & 10 & 33 \\
\hline 5 & May 2000 & LNA $1.60 \mathrm{~m}$ & Coudé & He I $6678 \AA$ & 5 & 56 \\
\hline 6 & April 2001 & ESO $1.52 \mathrm{~m}$ & FEROS & $3560-9200 \AA$ & 3 & 59 \\
\hline
\end{tabular}

$1.5,3.8$ and $5.3 \mathrm{c} / \mathrm{d}$, with the same time distribution as the 1996-2000 LNA data set. These spectra have been affected by random noise with a Gaussian distribution, whose $F W H M$ represents about $30 \%$ of total amplitude of the input signal. The frequencies used in this test were the same as those found in the actual spectra, and all the synthetic spectra were generated with phase-dependence along the wavelength bins. From this set, we constructed 241 time series formed at each $0.1 \AA$ across the line profile in the same way as we had done with real data. The series were then analyzed with the Cleanest algorithm with appropriate frequency step, of around $0.0005 \mathrm{c} / \mathrm{d}$, corresponding to the total time span. The resulting periodogram is shown in Fig. 2.

In order to discover whether the frequencies detected by this method are actually related to the star signal or whether they simply reflect the time sampling of data, we carried out to another test by shuffling the synthetic intensities at random, though taking care that the original time sampling was preserved. The resulting periodogram is shown in Fig. 3. A study of the frequency of detected signals in Fig. 3 shows that none is statistically significant. Further analyses taking different combinations of the data sampling also yielded similar results. This shows that all periods displayed in Fig. 2 are robust against random selection of the data points. On the other hand, as the signals displayed in the periodogram of Fig. 2 as well as the amplitude of the simulation noise are close to those of the real data, our results should not be strongly affected by the window spectrum.

\subsection{Results on Ipv}

LNA spectra were arranged in four main sets: 1996, 1997 to 1998,2000 and 1996 to 2000 . Each data set was divided into 241 time series, from $6664.9 \AA$ to $6688.9 \AA$ with steps of $0.1 \AA$. All series were analyzed with frequency steps in the range 0.2 to $0.0006 \mathrm{c} / \mathrm{d}$, depending on the sample to be studied. The lpv frequencies with highest significance $(\geq 75 \%$ confidence level) detected with Cleanest in all data sets concerning $\mathrm{He}_{\text {I, }}, \mathrm{Fe}$ II, Mg II and $\mathrm{Si}$ iI lines are shown in Table 2. Figure 4 shows the resulting periodogram for the 1997 to 1998 data. The confidence diagram resulting from a $\chi^{2}$ test for the periodicities found in the He $16678 \AA$ line is shown in Fig. 5. We can readily see that, besides a signal with $v=2.5 \mathrm{c} / \mathrm{d}$, frequencies greater than $6 \mathrm{c} / \mathrm{d}$ are of lower significance, so they are less trustworthy. The He I $\lambda 6678 \AA$ lpv was analyzed using only LNA spectra, since ESO spectra presented problems related to

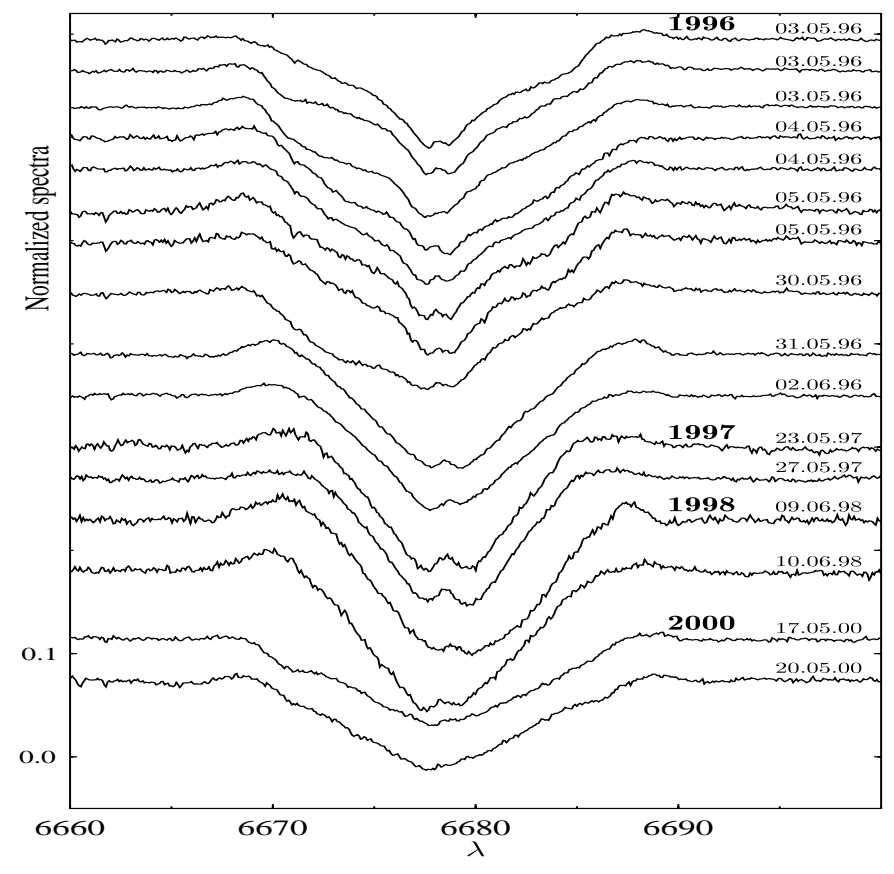

Fig. 1. Spectroscopic sampling of LNA spectra, centered on He r $6678 \AA$ line profile.

bad columns of CCD in that wavelength region. Results concerning lines other than He I $\lambda 6678 \AA$ correspond to the 20002001 epoch.

Figure 6 pictures in a grey scale the dynamic spectra of the pulsation cycles for He $\mathrm{I} \lambda 6678 \AA$ line. A total of 652 spectra from 1996 to 2000 were sorted. All spectra falling into the same phase bin were averaged to minimize the influence of other variabilities and noise (i.e. no prewhitening was applied). They are presented as residuals from the respective mean profiles and folded with frequencies $v_{2}$ (left), $v_{3}$ (center) and $v_{4}$ (right).

The signals with high degree of confidence are $0.61 \pm$ $0.05 \mathrm{c} / \mathrm{d}\left(v_{1}\right), 1.48 \pm 0.05 \mathrm{c} / \mathrm{d}\left(v_{2}\right), 3.81 \pm 0.28 \mathrm{c} / \mathrm{d}\left(v_{3}\right)$ and $5.31 \pm 0.19 \mathrm{c} / \mathrm{d}\left(v_{4}\right)$. The highest considered frequencies, $9.24 \pm$ $0.19 \mathrm{c} / \mathrm{d}\left(v_{5}\right)$ and $10.35 \pm 0.13 \mathrm{c} / \mathrm{d}\left(v_{6}\right)$ were found only in He I $\lambda 6678 \AA$. The uncertainty related to time sampling for these signals is of the order $\sim 0.05 \mathrm{c} / \mathrm{d}$, considering the averaged data span of 1996, 1997-1998 and 2000 sets, weighted by the number of observed spectra. A number of frequencies displayed in Table 2 are in agreement with our previous results for this star, like $1.48 \mathrm{c} / \mathrm{d}, 1.78 \mathrm{c} / \mathrm{d}, 5.31 \mathrm{c} / \mathrm{d}$ (Janot-Pacheco et al. 1999). As in Janot-Pacheco et al. (1999), we have also 


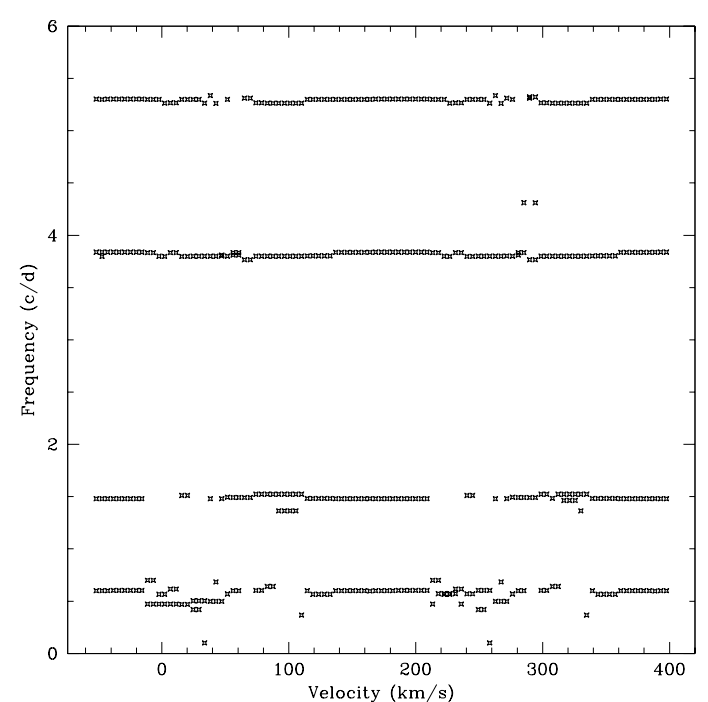

Fig. 2. Periodogram of synthetic residuals with time distribution (window spectrum) equal to that of LNA spectra, from 1996 to 2000.

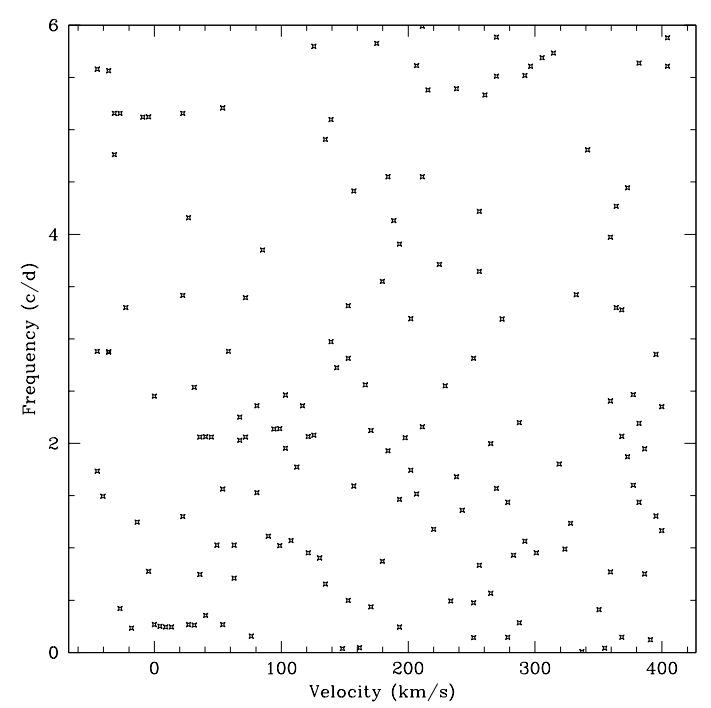

Fig. 3. Periodogram of synthetic residuals with the same time distribution as Fig. 2, but the input signals were randomly shuffled. Notice that none of the previous frequencies were found, suggesting that they are not strongly dependent on data sampling.

found a signal at $4.52 \mathrm{c} / \mathrm{d}$, but only in the $\mathrm{He}_{\mathrm{I}} \lambda 4471$ line. Since this signal can be an alias of $3.52 \mathrm{c} / \mathrm{d}$, we do not report it in Table 2 . The frequencies found in the $1 p v$ analyses ( $v_{2}$ to $v_{6}$ ) are attributed to NRP modes. The $0.61 \mathrm{c} / \mathrm{d}$ signal $\left(v_{1}\right)$ is discussed in Sect. 6, where it is shown that it is compatible with the presence of an ejected orbiting shell. In Sects. 4.1 and 4.2 it is shown that the $1.3 \mathrm{c} / \mathrm{d}$ signal can be associated with stellar rotation, since it was determined from the continuum and line spectra, and was also found in the lpv analyses of $\mathrm{He}$ I $\lambda \lambda 4026$, $4388 \AA$ and Si II $\lambda 4131 \AA$ whose detection could be assured through the presence of inhomogeneities such as spots (Balona 1990).

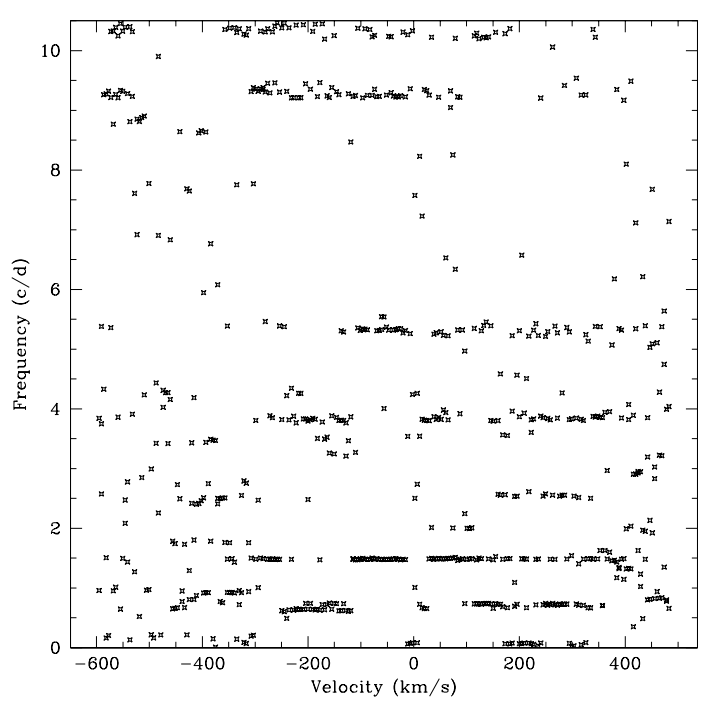

Fig. 4. Periodogram of He $\mathrm{I} 6678 \AA$ line profiles obtained at LNA in $1997 / 1998$.

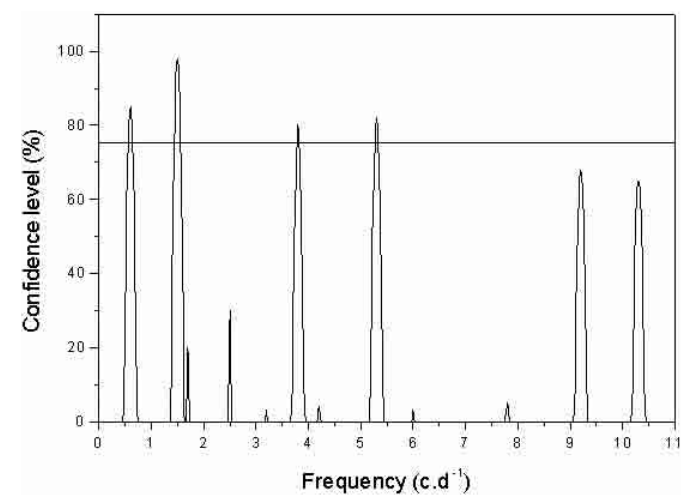

Fig. 5. Diagram of confidence level for periodicities found in He I $6678 \AA$ line profiles. The horizontal line indicates $75 \%$ confidence level.

\subsection{Global line profile variations}

Besides the $1 p v$ analyzed in the previous section, variabilities in radial velocity $(R V)$, equivalent width $(E W)$ and full width half maximum $(F W H M)$ measurements in He $\mathrm{I} \lambda 6678 \AA$ line profiles were also detected. Table 3 compares the frequencies obtained from these global line profile variations with those found in the previous section. Figure 7 shows the $R V, E W$ and FWHM variations of the He I 6678 line from 1996 to 2000 where the mid-term variation of the line profile can be seen. There is a noticeable anticorrelation between the $E W$ and the $F W H M$ of the line.

\subsection{Photometric variations}

Photometric data of HD 127972 from the Hipparcos satellite (ESA 1997) obtained from 1990 to 1992 were also analyzed. In this case, the time series analysis with Cleanest indicated a strong signal with frequency $v_{\text {phot }}=1.55 \mathrm{c} / \mathrm{d}$. The photometric data folded with this frequency are shown in Fig. 8. These data cannot be recast into a neat phase-dependent diagram with a lower frequency, in particular with $1.3 \mathrm{c} / \mathrm{d}$, since the amplitude 

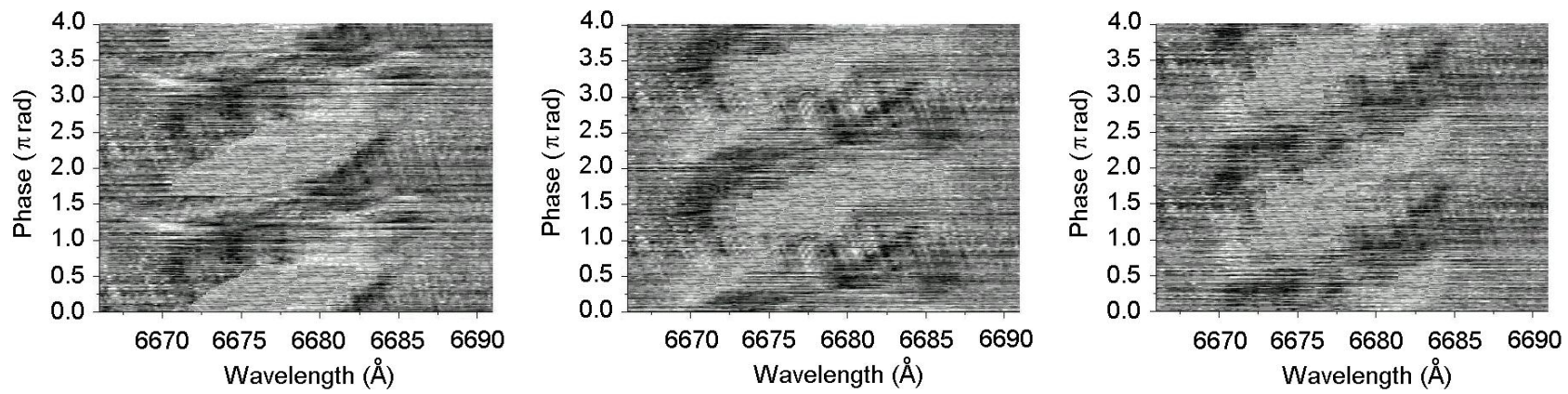

Fig. 6. Grey-scale of spectroscopic residuals centered at He I $6678 \AA$, folded with $v_{2}$ (left), $v_{3}$ (centre) and $v_{4}$ (right).

Table 2. Results of time series analysis in all profiles.

\begin{tabular}{|c|c|c|c|c|c|c|c|}
\hline \multirow[t]{2}{*}{ Line Profile } & \multirow[t]{2}{*}{ Epoch } & \multicolumn{4}{|c|}{ Detected frequencies $(\mathrm{c} / \mathrm{d})$} & \multirow[b]{2}{*}{$v_{3}$} & \multirow[b]{2}{*}{$v_{4}$} \\
\hline & & $v_{1}$ & & $v_{2}$ & & & \\
\hline Fe II $5169 \AA ̊$ & $4-6$ & 0.61 & & 1.51 & & & 5.33 \\
\hline He I $4026 \AA$ & $"$ & 0.62 & 1.30 & 1.48 & & & \\
\hline He I $4121 \AA$ & $"$ & & & 1.49 & & & \\
\hline Her $4144 \AA$ & $"$ & 0.58 & & 1.47 & 1.78 & & \\
\hline He I $4388 \AA$ & $"$ & & 1.28 & & & 3.52 & \\
\hline He I $4471 \AA$ & $"$ & 0.61 & & 1.48 & 1.79 & & \\
\hline He I $4922 \AA$ & $"$ & 0.57 & & 1.50 & 1.82 & 3.51 & \\
\hline $\mathrm{Mg}_{\text {II }} 4481 \AA$ & $"$ & 0.63 & & & 1.71 & & \\
\hline Si п् $4131 \AA$ & $"$ & 0.62 & 1.29 & 1.50 & 1.70 & 3.81 & \\
\hline He $16678 \AA$ & 5 & 0.58 & & 1.47 & 1.71 & 3.52 & 5.31 \\
\hline He I $6678 \AA$ & $2-3$ & 0.61 & & 1.48 & & 3.81 & 5.31 \\
\hline Her $6678 \AA ̊$ & 1 & 0.61 & & 1.48 & & 3.81 & 5.31 \\
\hline He $6678 \AA$ & 1 to 5 & 0.61 & & 1.48 & & 3.81 & 5.31 \\
\hline
\end{tabular}

of the photometric signal is much more significant than the last one. We argue that $1.55 \mathrm{c} / \mathrm{d}$ could be associated with NRPs rather than with stellar rotation (see Sect. 4.1).

\subsection{Characteristics of the NRPs}

An approach to infer the pulsational degree $\ell$ and the azimuthal order $|m|$ was proposed by Telting \& Schrijvers (1997a) with the intensity period search method (IPS). This method takes into account the phase variation across the line profile of a frequency and its first harmonic. It is mainly an empirical formulation based on analyses of phase diagrams derived from generated time series of absorption line profiles of a nonradially pulsating early-type star. For diagnostic purposes, using a Monte Carlo simulation these authors quantified the relation between $\ell$ and $\Delta \Psi_{0}$ (phase difference of main frequency), and that between $|m|$ and $\Delta \Psi_{1}$ (phase difference of its first harmonic) for spheroidal modes. They found that the fitted coefficients are remarkably stable throughout the parameter space.

From the stability of the coefficients they concluded that it is possible to derive good estimates for the pulsation parameters $\ell$ and $|m|$ by evaluating the phase differences across the line profile. The typical uncertainties on $\ell$ and $|m|$ by using the IPS method are estimated to be \pm 1 and \pm 2 , respectively. Considering the previous detected lpv frequencies $v_{2}, v_{3}, v_{4}, v_{5}$ and $v_{6}$ as due to NRP, their pulsation parameters thus derived are given in Table 4.
Since we did not find first harmonics with significant amplitudes for most frequencies in the He I $\lambda 6678 \AA$ line profile, it was not possible to calculate their $|m|$ values by this method. We attempted to do this only for $v_{4}$, whose detected harmonic was supposed to be $v_{6}$ (see Table 4 and Fig. 9). Figure 9 are shown the IPS diagrams for frequencies $v_{2}$ to $v_{6}$. The upper panels show the phase diagram across the $\mathrm{He}_{\mathrm{I}} 6678$ line profile and the lower panels present their respective amplitudes. Figure 9 shows clearly the asymmetrical aspect of the amplitude of signals corresponding to frequencies $v_{4}$ and $v_{6}$. The same phenomenon was also seen by Floquet et al. (2000) in EW Lac. The $10.35 \mathrm{c} / \mathrm{d}$ signal could perhaps be considered the first harmonic of $5.31 \mathrm{c} / \mathrm{d}$ (scenario A). However, its power distribution does not exhibit the same behavior over the entire line profile, as can be expected for two harmonics, even when there are nonadiabatic effects (Schrijvers \& Telting 1999). Thus, we also considered the possibility that the two signals are independent (scenario B). In scenario A, IPS analysis leads to a pulsational degree $l=5$ and order $|\mathrm{m}|=4$, while in scenario B we obtain for $v_{4}$ and $v_{6} l=5$ and $l=8$ respectively. It can also be seen from this figure that the signal $v_{4}$ is not symmetrical around the line center, which should not be the case for NRP. However, the occurrence of central quasi-emissions in He I $6678 \AA$ transition at the 1996-1998 epoch could be partially responsible for the assymmetry observed in $v_{4}$ around the line center.

\section{Fundamental parameters}

Once the lpv frequencies are obtained, it is also important to determine the fundamental parameters of $\eta$ Cen. This will allow us, on the one hand, to distinguish the periodicities associated with rotation from those related to NRP and, on the other hand, to determine the evolutionary state of the star.

\subsection{Parameters derived from the continuum spectrum}

To determine the fundamental parameters of HD 127972 we used its BCD spectrophotometric data (Chalonge \& Divan 1952). They were derived from 12 low resolution spectra observed at ESO (La Silla, Chile) in May 1978 with the "Chalonge spectrograph" (Baillet et al. 1973). The advantages of using this system for Be stars were widely discussed in Zorec \& Briot (1991). It provides observational parameters that describe the photospheric Balmer discontinuity (BD) of the star and which are free from interstellar extinction and circumstellar emission/absorption perturbations. As they concern stellar 


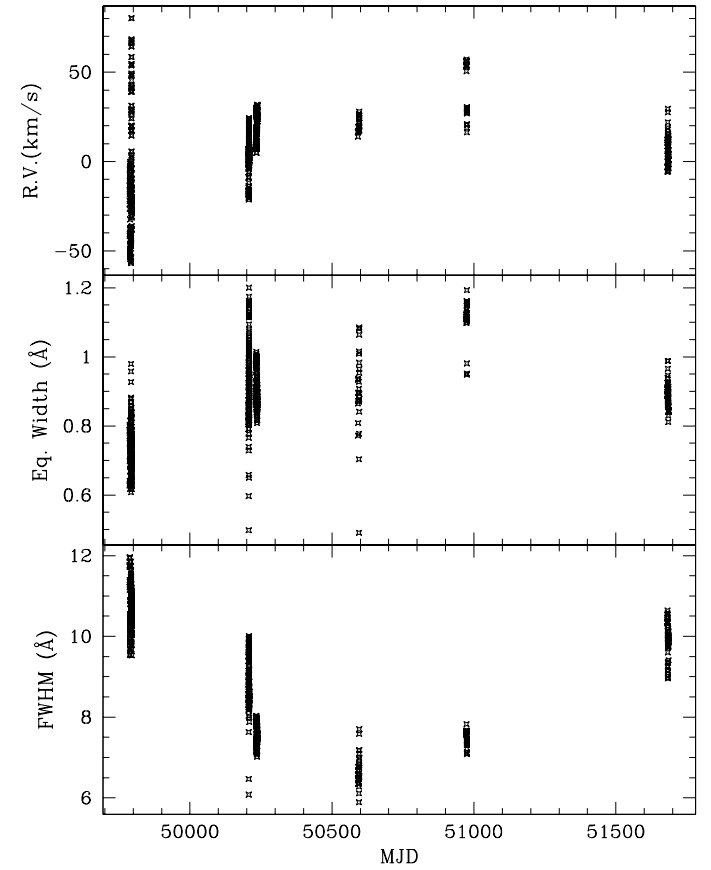

Fig. 7. Radial velocity $(R V)$, equivalent width $(E W)$ and $F W H M$ for all LNA spectra from 1996 to 2000.

Table 3. Comparative results among detected signals (c/d).

\begin{tabular}{lllll}
\hline \hline lpv's & $E W$ & $R V$ & $F W H M$ & $V / R$ \\
\hline 0.6 & 0.5 & 0.6 & 0.7 & 0.5 \\
1.5 & 1.6 & 1.6 & 1.5 & \\
3.8 & & 3.6 & & \\
5.3 & & & 5.0 & \\
9.2 & & & & \\
10.3 & & & & \\
\hline
\end{tabular}

layers where the continuum spectrum is formed, they originate in regions that can be assumed to be less perturbed by the stellar activities affecting the outermost atmospheric layers and commonly seen in spectral lines. The $\left(\lambda_{1}, D_{*}\right)$ parameters $\left(\lambda_{1}=\right.$ mean spectral position of the BD; $D_{*}=$ energy jump measured at $\lambda=3700 \AA$ ) represent the photospheric flux emitted by the observed stellar hemisphere, which in fast rotators is neither spherical nor has uniform surface temperature and gravity (Tassoul 1978). The emitted spectrum is then aspect angle dependent and may depict on average an object cooler and more evolved than it really is (Moss \& Smith 1981). Thus, the observed spectral and photometric parameters do not reflect the actual stellar mass and its evolutionary stage, if the observed quantities are interpreted simply using the current calibrations of fundamental parameters, as it the star were rotationless. Since the rotational energy of a rigid rotator is less than $1 \%$ of its gravitational energy (Zorec et al. 1988), we can assume that the evolutionary state of the star on the main sequence is described, in a first approximation, by models of non-rotating stars. So, to estimate the stellar mass and age from the usual evolutionary tracks, we need to determine the

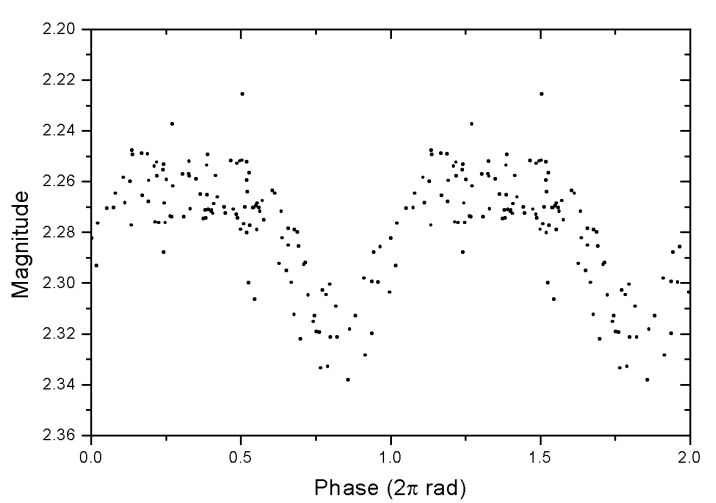

Fig. 8. Photometric Hipparcos light curve (ESA 1997) of HD 127972 folded modulo with its main detected frequency $(1.5 \mathrm{c} / \mathrm{d})$.

Table 4. Results of $\ell$ and $|m|$ parameters.

\begin{tabular}{llcc}
\hline \hline Frequencies & $\mathrm{c} / \mathrm{d}$ & $\ell( \pm 1)$ & $|m|( \pm 2)$ \\
\hline$v_{2}$ & 1.5 & 3 & \\
$v_{3}$ & 3.8 & 5 & \\
$v_{4} ; v_{6}$ & $5.3 ; 10.3$ & 5 & 4 \\
$v_{5}$ & 9.2 & 7 & \\
\hline
\end{tabular}

stellar rotationless parameters that underlie the observed ones. We disregarded subtle mixing effects on the stellar evolution produced by rotationally induced hydrodynamical instabilities (Endal \& Sofia 1979; Zahn 1983; Meynet \& Maeder 2000). We write then:

$\left.\begin{array}{l}L\left(\lambda_{1}, D_{*}\right)=L_{\mathrm{o}}(M, t) F_{L}(M, \omega, i, t) \\ D_{*}=D_{\mathrm{o}}(M, t) F_{D}(M, \omega, i, t) \\ \lambda_{1}=\lambda_{1}^{\mathrm{o}}(M, t) F_{\lambda_{1}}(M, \omega, i, t) \\ V \sin i=V_{\mathrm{c}}(M, t) \frac{R_{\mathrm{e}}(M, \omega, t)}{R_{\mathrm{c}}(M, t)} \omega \sin i\end{array}\right\}$,

where $L_{0}, D_{\mathrm{o}}$ and $\lambda_{1}^{\mathrm{o}}$ are respectively the bolometric luminosity, the BD and the $\lambda_{1}$ parameter of the star if it were rotationless; $F_{L}, F_{D}$ and $F_{\lambda_{1}}$ are functions calculated assuming the stars are rigid rotators. $M$ is taken as the "actual" stellar mass, $\omega=\Omega / \Omega_{\mathrm{c}}$ is the angular velocity ratio $\left(\Omega_{\mathrm{c}}\right.$ is the critical angular velocity), $i$ is the stellar aspect angle, $t$ is the stellar age, $V_{\mathrm{c}}$ is the critical linear equatorial velocity, $R_{\mathrm{c}}$ is the critical equatorial radius and $R_{\mathrm{e}}$ the "actual" equatorial radius at its rotational rate $\omega$. The function $F_{L}, F_{D}$ and $F_{\lambda_{1}}$ were obtained assuming a Roche model for the stellar deformation and von Zeipel's law for the effective temperature distribution, as previously done by many authors (cf. Maeder \& Peytremann 1970, 1972; Collins \& Sonneborn 1977; Collins et al. 1991). They were already used in Zorec \& Briot (1997), Floquet et al. (2000, 2002), Frémat et al. (2002), Zorec et al. (2002a,b).

The observed $\left(\lambda_{1}, D_{*}\right)$ parameters used to solve Eqs. (1) are given in Table 5. The corresponding apparent $\left(\log L / L_{\odot}, T_{\text {eff }}, \log g\right)_{\left(\lambda_{1}, D_{*}\right)}$ parameters obtained with calibrations suited to normal, rotationless B stars (Divan \& Zorec 1982) are also reproduced in Table 5. The observed bolometric luminosity $\log L / L_{\odot}$ introduced in (1) was estimated, however, using integrated fluxes over the entire spectrum and the Hipparcos parallax of $\eta$ Cen. The method used to obtain it also helps us to test the consistency of the 

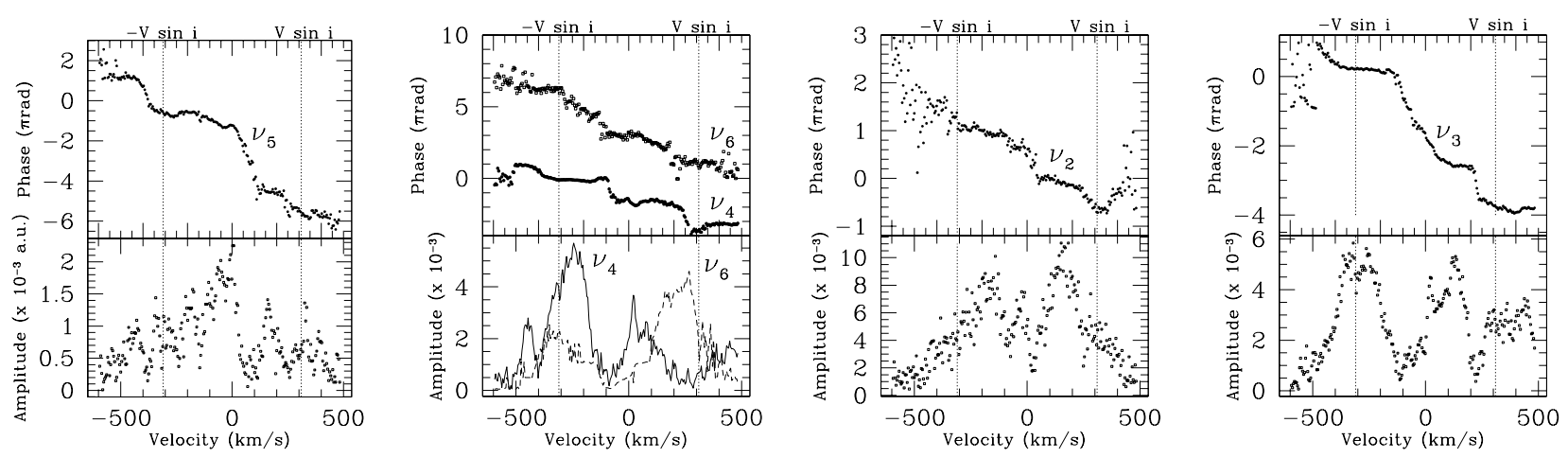

Fig. 9. IPS diagram for the detected lpv signals from $v_{2}$ to $v_{6}$. Upper panels show the phase diagram across the He I $6678 \AA$ line profile. Lower panels present their respective amplitudes. Vertical lines indicate $\pm V \sin i$.

$\left(\lambda_{1}, D_{*}\right)$-calibration dependent fundamental parameters. By definition the flux effective temperature of a star is:

$T_{\mathrm{eff}}^{4}=\frac{4}{\sigma_{\mathrm{SB}}} \frac{f}{\theta^{2}}$

where $\theta$ is the angular diameter of the star, in radians, $f$ is the flux received on earth, corrected from ISM extinction and integrated over the full extent of the spectrum and $\sigma_{\mathrm{SB}}$ is the Stefan-Boltzmann constant. The angular diameter of the star is given by:

$\theta=2\left(f_{\lambda} / F_{\lambda}\right)^{1 / 2}$

where $f_{\lambda}$ is the absolute monochromatic flux received on earth, corrected from ISM extinction, and $F_{\lambda}$ is the absolute monochromatic flux emitted in the star. $f$ was calculated using: 1) the 13-color photometry of Johnson \& Mitchell (1975) calibrated in absolute fluxes; 2) the UV flux observed by IUE in 1982 and TD-1 satellite. The 13-color photometry of $\eta$ Cen apparently corresponds to a stellar non-emission phase, as it can be controlled by its apparent magnitude $V\left(V_{\mathrm{o}}=2.24 \pm 0.03 \mathrm{mag}\right.$ dereddened; $E(B-V)=0.030 \pm 0.01 \mathrm{mag})$ and the $\mathrm{BD}$ $D_{*}=0.161 \pm 0.013$ dex obtained from the 13 -color absolute fluxes. The wavelength interval used to derive $\theta$ is $\lambda \lambda 0.5$ to $0.7 \mu \mathrm{m}$. The fluxes $F_{\lambda}$ and those employed to complete $f$ in the non-observed spectral region are from the non-rotating normal stellar atmosphere models of Kurucz (1994). Adopting the $\log g\left(\lambda_{1}, D_{*}\right)$ parameter, we iterated relations (2) and (3) until a difference $\Delta T_{\text {eff }}=1 \mathrm{~K}$ was attained between two consecutive steps. The values of $T_{\text {eff }}, f$ and $\theta$ thus obtained are given in Table 5. From the Hipparcos parallax of $\eta$ Cen we have its distance $d_{\text {HIPP }}=94.6_{-6.9}^{+8.1} \mathrm{pc}$ that from $f$ leads to the estimate of the stellar bolometric luminosity $\log L^{f} / L_{\odot}$ used in this work. With $\theta$ we can also obtain the stellar radius $R^{f} / R_{\odot}$. These parameters are also given in Table 5. It is worth noting the close resemblance of the bolometric luminosities, temperatures and radii obtained using these two completely different methods.

For comparison, let us quote the fundamental parameters of $\eta$ Cen recently determined by some authors. Harmanec (2000) obtained $T_{\text {eff }}=22400 \mathrm{~K}, \log L / L_{\odot}=3.876, R_{*} / R_{\odot}=5.7_{-0.4}^{+0.5}$ and $M=9 M_{\odot}$. As compared with our determination, the slightly higher value of $\log L / L_{\odot}$ obtained by Harmanec (2000) could be due to its $T_{\text {eff }}$ determination, which carries a difference of $0.23 \mathrm{mag}$ in the bolometric correction (BC) used
(Code et al. 1976). On the other hand, Code's et al. (1976) were calculated from the OAO-2 satellite far-UV energy measurements, which are overestimated as compared to those obtained since then with TD1 and IUE satellites. They may endanger the reliability of BC estimates for hot stars. The difference noticed in $T_{\text {eff }}$ might be due to the use of $u b v y \beta$ Strömgren's photometry and the calibrations by Moon \& Dworetsky (1985), which can lead to systematic deviations in the estimation of fundamental parameters (Frémat \& Zorec 2002). Štefl et al. (1995) obtained $T_{\text {eff }}=21860 \pm 480 \mathrm{~K}$ also using $u b v y \beta$ photometry, but with Napiwotzki's et al. (1993) calibration. The use of photometric data can easily lead to overestimated effective temperatures, mostly for hot Be stars, because even a slight $\mathrm{CE}$ emission, which is difficult to clear up, can affect the $u$ magnitude. Štefl et al. (1995) obtained $T_{\text {eff }}=21100 \pm 1370 \mathrm{~K}$ by fitting spectral lines with model atmospheres. This value is no far from the temperature we derived by a similar method (Sect. 4.2), but which we did not adopt for the present study. The stellar mass and radius determined by Harmanec (2000) are similar to those we inferred in this paper, but neither the starting $T_{\text {eff }}$ and $\log L / L_{\odot}$ parameters nor the methods used to obtain them are the same in the two attempts.

The system (1) was solved using a Monte Carlo method for the trials of the input set of parameters $\left(\log L / L_{\odot}, D_{*}, \lambda_{1}, V \sin i\right)$. Each parameter, $X$, was sampled in turn between two extreme values, $X \pm \Delta X$ where the amplitudes $\Delta X$ are quoted in Table 5 . We discarded about $10 \%$ of the solutions obtained, those which lead to $\omega>1$ and/or $\sin i>1$. The average values of reliable solutions of (1) derived from the remaining $90 \%$ trials are given in Table 5. The quoted dispersions do not represent errors, but the range of acceptable solutions. The relations between luminosities, masses and stellar ages used are from the evolutionary tracks of non-rotating stars calculated by Schaller et al. (1992) for $Z=0.02$.

Noting that $V \sin i$ is a key parameter to solve relations (1) and that the difference between Chauville's et al. (2001) and Slettebak's (1982) determinations is of the same order as their uncertainties, we sought the higher $V \sin i$ that, together with the above quoted uncertainties, still leads to reliable solutions of (1). We thus obtained $V \sin i=370 \mathrm{~km} \mathrm{~s}^{-1}$. Solutions presented in Table 5 encompass the input $V \sin i$ values centered once on $V \sin i=310 \mathrm{~km} \mathrm{~s}^{-1}$ that range from 266 to $354 \mathrm{~km} \mathrm{~s}^{-1}$ 
and then values centered on $V \sin i=370 \mathrm{~km} \mathrm{~s}^{-1}$ ranging from 326 to $414 \mathrm{~km} \mathrm{~s}^{-1}$.

Finally, we calculated the rotational frequency $v_{\mathrm{r}}=$ $0.02[V \sin i / \sin i]) /\left[R_{\mathrm{e}}(\omega) / R_{\odot}\right] \mathrm{c} / \mathrm{d}$, also given in Table 5, which can be compared with those obtained from time series analysis of lpv. They are, however, average values of only 27 determinations from the possible combinations of ( $V \sin i \pm$ $\left.\Delta_{V \sin i}, i \pm \Delta_{i}, R_{\mathrm{e}} \pm \Delta_{R_{\mathrm{e}}}\right)$ parameters, where each parameter takes in turn three values $X-\Delta X, X$ and $X+\Delta X$. The frequency derived from lpv time series analysis that more closely resembles $v_{\mathrm{r}}$ is $v=1.3 \mathrm{c} / \mathrm{d}$. A frequency $v=1.29 \mathrm{c} / \mathrm{d}$ was actually observed by Janot-Pacheco et al. (1999). Finally, we note that not only the rotational frequency $v_{\mathrm{r}}$ is far from the "critical" rotational frequency $v_{\mathrm{c}}$, which ranges from $1.34 \mathrm{c} / \mathrm{d}$ to $1.39 \mathrm{c} / \mathrm{d}$, depending on the value of $V \sin i$ adopted, but that it only marginally approaches the frequency $v=1.5 \mathrm{c} / \mathrm{d}$ suited for some $\mathrm{lpv}$ and the photometric variations.

Since for a star with mass $M \sim 8.6 M_{\odot}$ the main sequence (MS) life time is $t_{\mathrm{MS}} \simeq 3.0 \times 10^{7} \mathrm{yr}$, the ages estimated imply that $\eta$ Cen displays the Be phenomenon at $t \sim 0.6 t_{\mathrm{MS}}$, roughly the earliest epoch at which this phenomenon seems to appear in open clusters (Fabregat \& Torrejón 2000). Only if we adopted the observed fundamental parameters $\left(T_{\text {eff }}, L / L_{\odot}\right)$ without correcting them from rotational effects would the star apparently be at the end of its MS life $\operatorname{span}\left(t_{\text {app }}=2.8 \times 10^{7} \mathrm{yr}\right)$, as expected from theoretical predictions (Maeder \& Meynet 2000). In these models, the high stellar rotation and hence, the Be phenomenon, are meant to appear as the consequence of the evolution of the stellar internal rotational law.

\subsection{Parameters derived from the line spectrum}

Unfortunately the only spectra we have at our disposal of HD 127972 covering a large spectral range are for the years 2000 and 2001, when this star displayed well developed $\mathrm{H} \alpha$ emission. In spite of the fact that the estimation of fundamental parameters from these spectra brings in the influence of the CE, it can in principle furnish an extreme limit for these values. We calculated a grid of models with $T_{\text {eff }}$ and $\log g$ around the values displayed in Table 5 with steps $\Delta_{T_{\text {eff }}}=200 \mathrm{~K}$ and $\Delta_{\log g}=0.1 \mathrm{dex}$ using the codes of non LTE model atmospheres TLUSTY (Hubeny 1988) and SYNSPEC (Hubeny et al. 1994) to synthesize line profiles. Fundamental parameter determination was attempted employing Wolf's (1973) method with a $\chi^{2}$ controlled fit of $\mathrm{H} \gamma$ and $\mathrm{H} \delta$ absorption lines combined with reproduction of the Si II $\lambda 4131 / \mathrm{Si}$ III $\lambda 4553$ and He I $\lambda 4388 / \mathrm{He}$ iा $\lambda 4686$ equivalent width ratios. The results are summed up in Fig. 10. From this figure it seems that from the spectral lines used, the stellar parameters are $T_{\text {eff }}=21600 \pm$ $960 \mathrm{~K}$ and $\log g=3.34 \pm 0.16$ dex (marked " $\mathrm{x}$ " in Fig. 10).

The difference between the solutions attempted for the $\mathrm{H} \gamma$ and $\mathrm{H} \delta$ lines is explained by a different amount of line filling in with circumstellar emission. It can also be noted that $\mathrm{H} \delta$ is not clean from this emission. In both cases the residual emission leads to a lower gravity and to a hotter stellar temperature estimation.

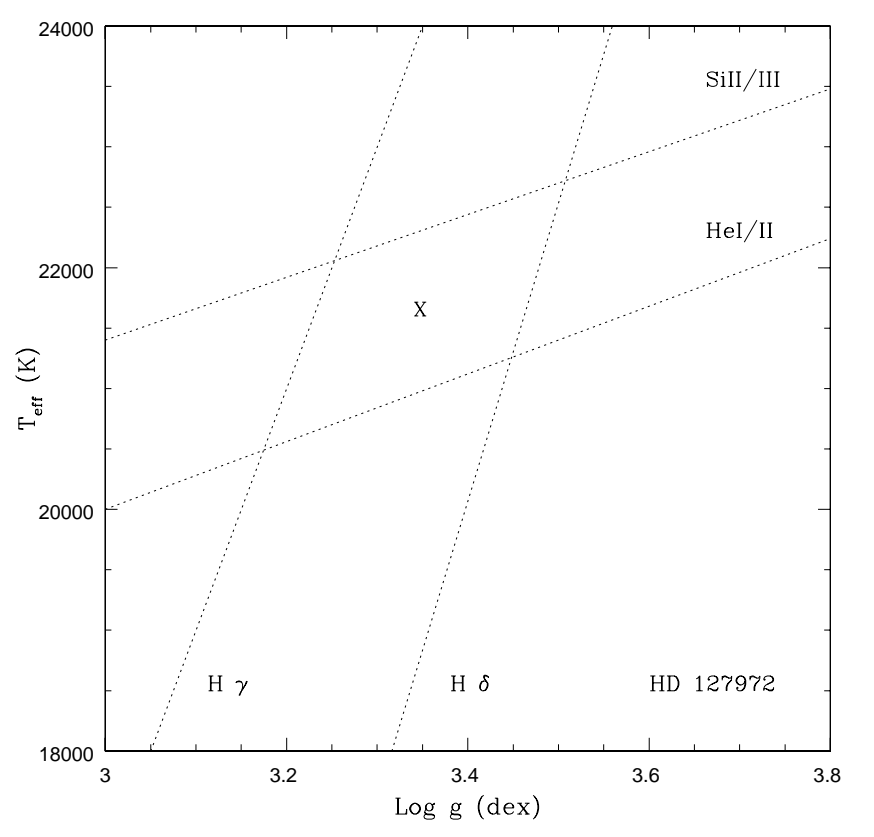

Fig. 10. $T_{\text {eff }}$ and $\log g$ solutions from fitting of the observed $\mathrm{H} \gamma$ and $\mathrm{H} \delta$ line with predicted profiles by non-LTE model atmospheres and model representation of $\mathrm{Si}$ II $\lambda 4131 / \mathrm{Si}$ III $\lambda 4553$ and $\mathrm{He}$ I $\lambda 4388 / \mathrm{He}$ II $\lambda 4686$ equivalent width ratios.

Notice the difference between the solutions obtained for the $\mathrm{Si}$ and $\mathrm{He}$ equivalent width ratios. Both series of lines are currently assumed to be little affected by circumstellar emission/absorption. On the other hand, according to von Zeipel's theorem, the local effective temperature of $\eta$ Cen ranges from $22700 \mathrm{~K}$ at the pole to $19900 \mathrm{~K}$ at the equator. As equivalent widths of the studied lines are increasing functions of $T_{\text {eff }}$ in this range of temperatures, the difference between solutions for the $\mathrm{Si}$ and $\mathrm{He}$ line ratios means that the stellar polar region more strongly favors the radiation fluxes in the $\mathrm{Si}$ than in He lines. This selection effect on formation region is also seen in the $V \sin i$ estimates, since from the $\mathrm{Si}$ we obtain $(V \sin i)_{\mathrm{Si}}=250 \mathrm{~km} \mathrm{~s}^{-1}$, while from He lines it assumes $(V \sin i)_{\mathrm{He}}=310 \mathrm{~km} \mathrm{~s}^{-1}$. This fact warns against the use of photospheric lines for $V \sin i$ determinations in fast rotators whose intensity increases with higher temperatures, since this parameter may result in underestimation.

\section{Line activity}

\subsection{Observations}

\subsection{1. $\mathrm{H} \alpha$ line}

HD 127972 showed $\mathrm{H} \alpha$ emission intensity strengthening from 1996 through 2000 and a slight fading in 2001; this last was accompanied by increased central absorption. The average $\mathrm{H} \alpha$ emission line profiles observed in the 1996, 1997, 1998, 2000 and 2001 epochs are shown in Fig. 11. This pattern of $\mathrm{H} \alpha$ line profiles variation resembles that displayed by the star in the 1987-1993 period (Hanuschik et al. 1996), but in the opposite sense. Putting both patterns together, a kind of cyclic emission variation of about 6-7 years appears. 
From $34 \mathrm{H} \alpha$ emission line profiles obtained from Mar. 12 to Mar. 23, 2000, a rapid cyclic variability of the $V / R$ emission peak intensity ratio (Fig. 12) and a rough increase, followed by a rapid decrease in the separation of the emission peaks, was observed (Fig. 13). The total peak separation change is not greater than $15 \mathrm{~km} \mathrm{~s}^{-1}$. Let us note also that the highest peak separation corresponds to the highest $V / R$ value. After this maximum, the $V / R$ ratio and the peak separation decreased. These changes were accompanied by a general wiggling of the line emission profile, characterized by a noticeable pulling down of the whole blue emission wing that produced the observed $V / R$ decreasing ratio. This drop of the emission intensity on the blue side of the emission line profile was followed by an increase of the red wing, but of much smaller amplitude. The maximum intensity changes in the blue wing were $\Delta I \sim 0.1$ ( $I$ is the intensity normalized to the local continuum) and they were produced in the spectral region from $V \sim-350$ to $-800 \mathrm{~km} \mathrm{~s}^{-1}$, while the intensity variation in the remaining profile was $\Delta I \sim 0.05$. Transient sharp absorption spikes were also observed far out in the wings.

\subsubsection{He 6678 line}

Each epoch corresponding to four season-averaged $\mathrm{H} \alpha$ emission line profiles shown in Fig. 11 is also characterized by a different spectral extent of the He I 6678 lpv. Figure 14 displays the mean absolute deviations of the lpv across the He I 6678 line profile, as defined by Walker (1991). It can be seen in this figure that the wavelength interval showing profile variability systematically widens from 1996 through 2000. The wavelength interval where the variability of highest significance extends beyond the $V \sin i$ limit, from roughly $\pm 390 \mathrm{~km} \mathrm{~s}^{-1}$ in 1997 to about $\pm 450 \mathrm{~km} \mathrm{~s}^{-1}$ in 2000. This behavior was also observed in other Be stars and in particular in $\lambda$ Eri by Kambe et al. (1993 and references therein).

Regarding the uncertainties in the determination of $V \sin i$ we can adopt, using the data in Table $5, V_{\mathrm{c}} \sin i=$ $490 \sin 66^{\circ} \mathrm{km} \mathrm{s}^{-1}$ as the reference value for the projected critical rotational velocity. Then, the line activity that extends up to $\pm 450 \mathrm{~km} \mathrm{~s}^{-1}$ could imply that the stellar layers where the He 6678 line is formed may be in critical rotation. However, this rotation is somewhat puzzling, because from the He 1471 line observed in April and May 2000 we obtained $V \sin i=310 \mathrm{~km} \mathrm{~s}^{-1}$ (Sect. 4.2). At least two explanations can be put forward for this discrepancy: 1) the main contributions to He I 6678 and He 4471 lines come respectively from different atmospheric layers [as expected from the ratio of their respective oscillator strengths $f(6678) / f(4471)=5.6]$, so that while the uppermost layers are accelerated to the critical rotational velocity, the lower ones would be left unscathed; 2) the contribution to the He I 6678 line wings at velocities $V>|V \sin i|$ is due to exophotospheric or circumstellar material travelling at velocities up to $\left(v_{\text {radial }}^{2}+v_{\text {tangential }}^{2}\right)^{1 / 2} \gtrsim 450 \mathrm{~km} \mathrm{~s}^{-1}$. The first possibility recalls the scenario proposed by Osaki (1986) and Saio (1994), where the prograde NRP modes accelerate the outer stellar atmospheric equatorial layers up to the critical rotational velocity. These layers may give rise to mass loss and
Table 5. Fundamental parameters of $\eta$ Cen.

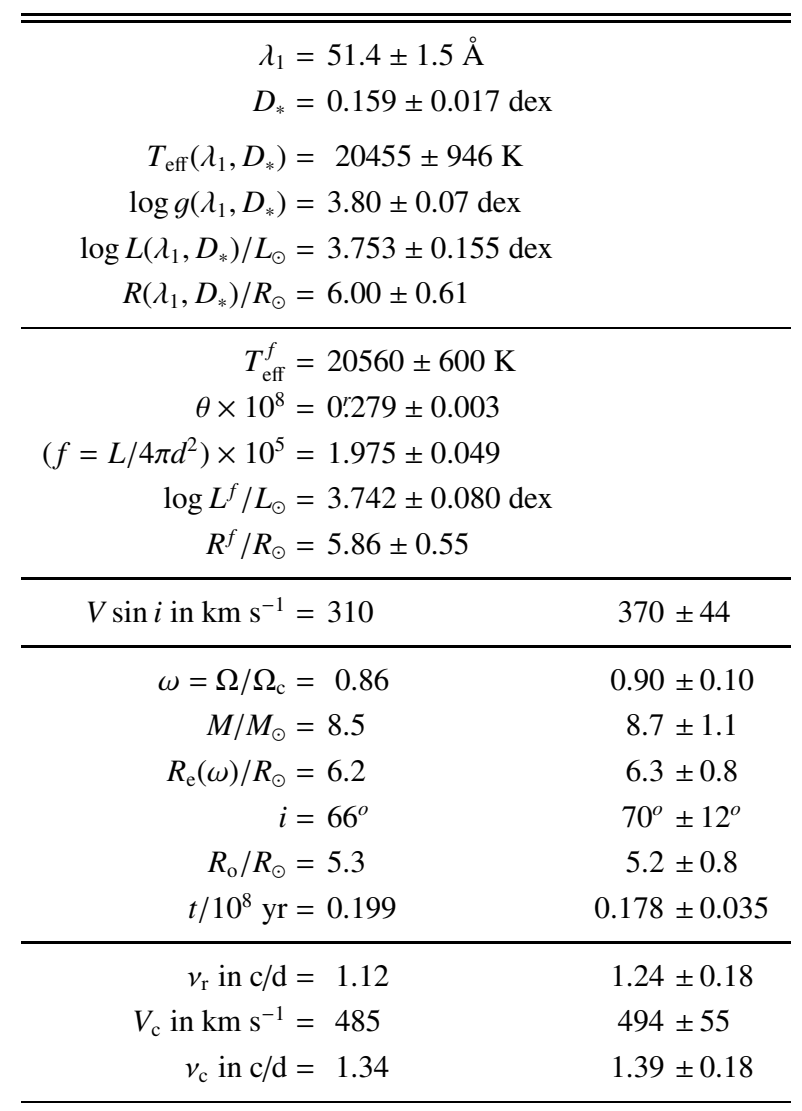

the NRP modes decrease in intensity as the mass dissipates in the extended envelope.

We studied the He $6678 \mathrm{lpv}$ beyond the $V \sin i$ limit. In order to obtain the characteristic frequencies, the red and the blue sides of the line profiles were folded into a unique positive velocity scale. The periodograms that resulted for the periods 1996, 1997/1998 and 2000, and the respective diagrams of confidence levels of the signals found are shown in Fig. 15. In the confidence level estimations we excluded the points at $V<310 \mathrm{~km} \mathrm{~s}^{-1}$. Since roughly in the interval \pm 400 to $\pm 500 \mathrm{~km} \mathrm{~s}^{-1}$ the emission shoulders seen in the line profiles are probably formed in the circumstellar regions, we can consider frequencies $v<v_{\mathrm{r}}$ as due to circumstellar orbiting matter. If so, $v=0.1 \pm 0.1 \mathrm{c} / \mathrm{d}$ could represent a perturbation produced at distances 3.3 to $6.4 R_{*}$ away from the star, depending on whether we consider Keplerian or angular momentum conservation rotation law respectively. From the diagrams of Fig. 15 we see that the signal $v=0.1 \pm 0.1 \mathrm{c} / \mathrm{d}$ disappears gradually from 1996 to 2000 , as it would be overtaken by matter gathered elsewhere and with a more significant contribution to the line profile. On the contrary, the signal $v=0.6 \pm 0.1 \mathrm{c} / \mathrm{d}$ is ubiquitous and its significance grows from 1996 to 2000 (the confidence peaks must be regarded relative to each other only within a given diagram). It may correspond to perturbations centered from 1.3 to $2.0 R_{*}$ in the $\mathrm{CE}$, depending on the rotational law assumed. As emission in the $\mathrm{H} \alpha$ line also grows from 1996 to 2000, we may consider there is an increasing amount of matter gathered in these $\mathrm{CE}$ regions. This picture seems to be confirmed by an 


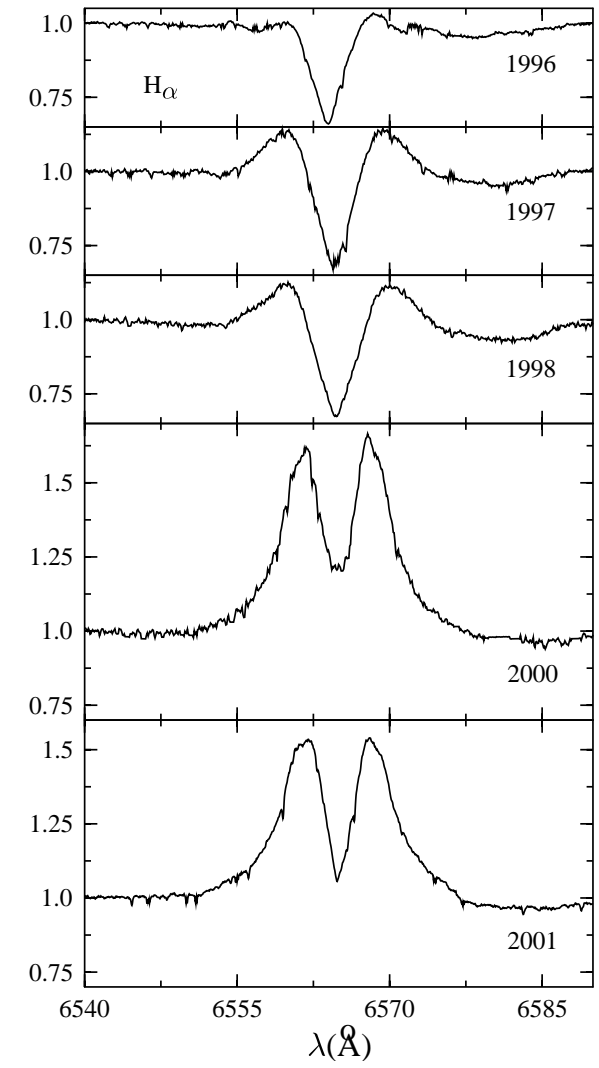

Fig. 11. Average $\mathrm{H} \alpha$ line profiles for the 1996, 1997, 1998, 2000 and 2001 epochs.

increasing CE density derived using a simple model of the $\mathrm{H} \alpha$ line emission presented in Sect. 5.2. The widening of the wavelength interval of $\mathrm{lpv}$ in the He I 6678 line could be then associated with a period of increased mass ejection.

There is also the prevalent frequency $v=1.5 \pm 0.1 \mathrm{c} / \mathrm{d}$ that could be due to the central star, which as seen in Sect. 3.2. is quite outstanding in the data analyzed in the present paper.

Let us finally note that if $V \sin i$ actually changed from 310 to $450 \mathrm{~km} \mathrm{~s}^{-1}$, this would represent a variation of only $14 \%$ in $\Omega / \Omega_{\mathrm{c}}$, while the $45 \%$ variation of $V \sin i=R_{\mathrm{e}}(\Omega) \Omega \sin i$ is provided mainly by rotational stretching of the equatorial radius $R_{\mathrm{e}}(\Omega)$.

\subsection{Modeling}

One of the most difficult questions relating to Be stars concerns their CE formation. It is then important to determine the relevant parameters that characterize the $\mathrm{CE}$ structure at each observed emission phase. This may help us to estimate the effects of stellar activity on the observed CE changes. In particular, if the apparently increasing activity detected in the He $\mathrm{I} 6678$ line from 1996 to 2000 also implied conspicuous mass ejections, the average density of the CE must have changed perceptibly, so that we can detect it by studying the emission in the $\mathrm{H} \alpha$ line. In order to obtain a rough insight on the scale factors characterizing the CE structure, we use first physical principles and a simple representation of the envelope. From Sect. 4.2 it seems that $\eta$ Cen is seen nearly equator-on. We assume then that the $\mathrm{CE}$ is represented by a rotating cylindrical disc seen edge-on. It can also be simultaneously expanding or contracting. Since the main radiation transfer effects are controlled by the optical depth, which is an integrated quantity, the disc can be treated in a first approximation as a rotating/expanding (or contracting) ring with the same radial optical depth as the CE is thought to have (Floquet et al. 2000). The ring has a radius $R$ and a total height $h=2 \times H$.

It has long been known that the source function $S_{\mathrm{H} \alpha}$ of the $\mathrm{H} \alpha$ line in B stars is strongly dominated by radiative ionization and recombination processes (Thomas 1965; Jefferies 1968). In a slab, as the one represented by the ring facing the central star, we can then use the following dependence of the source function with the optical depth (Mihalas 1978):

$S_{\mathrm{H} \alpha}\left(\tau_{\mathrm{o}}\right)=\left\{\begin{array}{r}\eta^{1 / 2} B^{*} \text { for } \tau_{\mathrm{o}} \leq 1 \\ \eta^{1 / 2} B^{*} \tau_{\mathrm{o}}^{1 / 2} \text { for } \tau_{\mathrm{o}}>1,\end{array}\right.$

where $\tau_{\mathrm{o}}$ is the optical depth at the center of the $\mathrm{H} \alpha$ transition. For radiative fields around $\mathrm{B}$ stars, $\mathrm{CE}$ electron temperatures of the order of $T_{\mathrm{e}} \sim 0.8 \times T_{\text {eff }}$ and electron densities $N_{\mathrm{e}} \lesssim$ $10^{13} \mathrm{~cm}^{-3}$, it can be shown that:

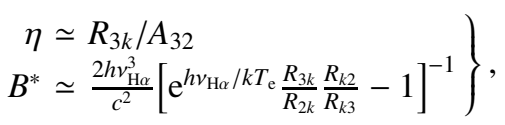

where $R_{n k}$ and $R_{k n}$ are the radiative ionization and recombination rates to the $n$-atomic level respectively and $A_{32}$ is the spontaneous emission rate.

The wavelength-dependent $\mathrm{H} \alpha$ line optical depth was assumed to be:

$\tau_{\lambda}=\tau_{\mathrm{o}} \Phi(\Delta \lambda)$

where $\Phi$ is the Voigt function given in the approximation suggested by Dobrichev (1984) and recommended by Piskunov (1992). The wavelength displacement $\Delta \lambda$ is produced by the total velocity of the ring projected along the line of sight $\pm \mu V_{\mathrm{r}} \pm\left(1-\mu^{2}\right)^{1 / 2} V_{\Omega}[\mu=\cos$ (radial direction, line of sight) $]$ where the signs are chosen according to the sector of the ring facing the observer and whether it concerns the front or rear part of the ring.

From (5) we see that the radiation field of the underlying star determines the value of the source function, so that $\eta^{1 / 2} B^{*} \simeq 0.05$. The rotationally broadened photospheric absorption line profile is obtained using the flux $\mathrm{H} \alpha$ calculated for $V \sin i=0 \mathrm{~km} \mathrm{~s}^{-1}$ from Kurucz' codes and using the fundamental stellar parameters presented in Table 5. The fit of each observed mean $\mathrm{H} \alpha$ line profile (Fig. 11) is then obtained using $R, H, V_{\mathrm{r}}, V_{\Omega}$ and $\tau_{\mathrm{o}}$ as free parameters. $V_{\mathrm{r}}$ determines the eventual asymetry seen in the emission peaks. It was determined only for the 1996 line profile; in other cases we considered $V_{\mathrm{r}}=0 \mathrm{~km} \mathrm{~s}^{-1}$. The separation of the emission peaks is determined mainly by $V_{\Omega}$, but it also depends on $\tau_{\mathrm{o}}$. The full width of the emission line on its half intensity and on the low side of wings approaching the continuum level is fixed by $R$ and $\tau_{\mathrm{o}}$. For a given value of $R, H$ and $\tau_{\mathrm{o}}$ determine the emission intensity in the peaks. The ratio $H / R$ and $\tau_{\mathrm{o}}$ establish the depth of the central absorption. The fits thus obtained are shown in Fig. 16 (dashed lines) and the corresponding CE parameters 


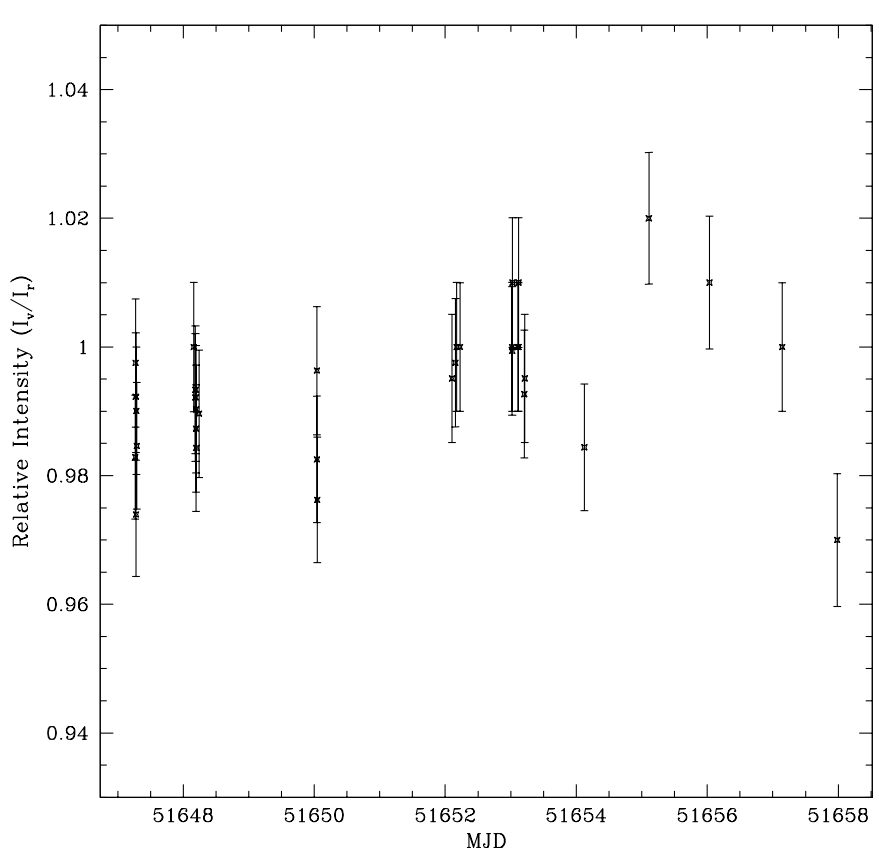

Fig. 12. $\mathrm{H} \alpha V / R$ ratio between Mar. 12 and Mar. 23, 2000.

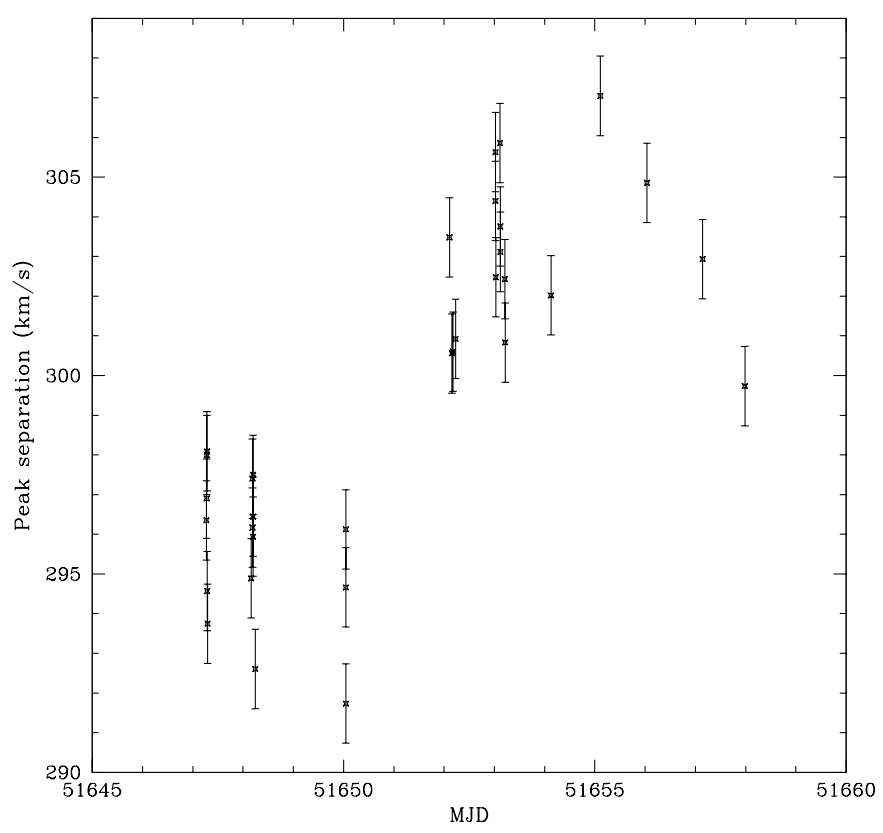

Fig. 13. H $\alpha$ peak separation between Mar. 12 and Mar. 23, 2000.

are given in Table 6 . We considered the $1998 \mathrm{H} \alpha$ emission line profile as essentially the same as in 1997 , so we did not produce a fit for this line.

\section{Discussion}

In our previous work (Janot-Pacheco et al. 1999), a signal with $0.78 \mathrm{c} / \mathrm{d}$ was detected and interpreted as due probably to a NRP mode. Keeping in mind the mechanism of mass ejection based on prograde modes proposed by Osaki (1986), such a signal is not expected to produce mass ejection, as it is retrograde. A similar signal was not detected in this work, but another was found with $0.6 \mathrm{c} / \mathrm{d}$. According to the fundamental stellar

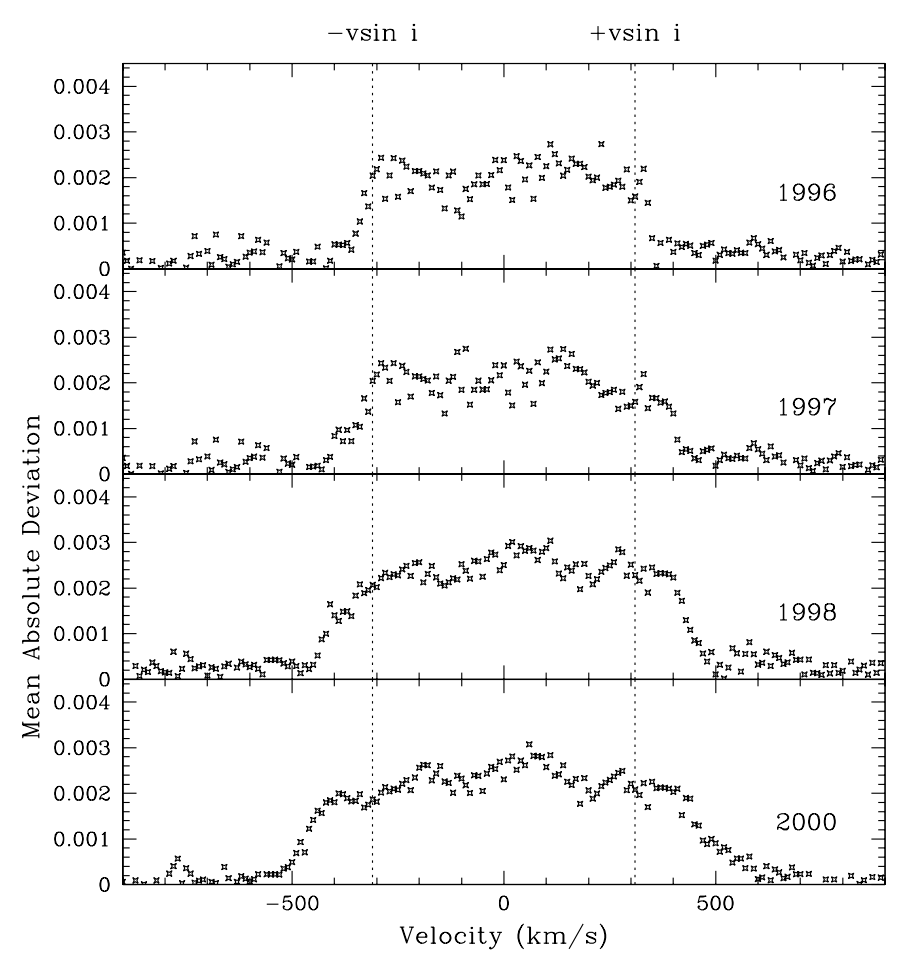

Fig. 14. Time evolution of mean absolute deviations of line profile variations detected in He I $\lambda 6678 \AA$ in four periods, 1996, 1997, 1998 and 2000. Notice the increase of variability beyond the limits of $V \sin i$ from 1996 to 2000, in apparent correlation with the strengthening of emission in the $\mathrm{H} \alpha$ line.

parameters presented in Table 5 , the frequency $v_{1}=0.6 \mathrm{c} / \mathrm{d}$ can also be interpreted as being due to an orbiting perturbation either at $R / R_{*} \simeq 2.0$ for a Keplerian velocity law or at $R / R_{*} \simeq 1.3$ if an angular momentum conservation law is prevailing.

For comparison, we give in Table 6 the semi-separation of emission peaks $V_{\mathrm{P}}$ (in $\mathrm{km} \mathrm{s}^{-1}$ ) in the $\mathrm{H} \alpha$ line profiles and the radius $\left(R / R_{*}\right)_{\mathrm{K}}$ associated with $V_{\mathrm{P}}$ assuming the $\mathrm{CE}$ is in Keplerian rotation. We see that $R / R_{*} \sim(1.7 \pm 0.3)\left(R / R_{*}\right)_{\mathrm{K}}$ while $V_{\Omega} \sim(1.5 \pm 0.3) V_{\mathrm{P}}\left(V_{\Omega}\right.$ and $V_{\mathrm{P}}$ are both aspect angle projected velocities). This peculiar behavior is due to the $\mathrm{CE}$ opacity effect (Hummel 1994; Chauville et al. 2001), according to which for line profiles of 1996-1998 type our simulations show that keeping the parameters $\left(V_{\Omega}, R, H\right)$ unchanged, the line emission peak separation widens as $\tau_{\mathrm{o}}$ increases from $\tau_{\mathrm{o}}=0$ to 1.0. It stretches down again for still higher values of $\tau_{o}$. In line profiles of 2000-2001 type, the separation of the emission peaks reduces for increasing values of $\tau_{\mathrm{o}}$. Due to this effect, it is difficult to infer from the present numerical simulation the characteristics of the CE rotational law. Such a deduction is further hindered by the fact that $V_{\Omega}$ is an average rotational velocity that probably resumes the kinematic characteristics of the whole CE from the star up to an external radius $R_{\mathrm{E}} / R_{*} \gg R / R_{*}$. Let us still note that for $\tau_{\mathrm{o}} \gg 1$, because of the $\tau_{\mathrm{o}}^{1 / 2}$-dependence of the source function, our model line profiles become bottle-shaped.

Noting that for the sake of a simple order of magnitude estimation we can write $\tau_{\mathrm{o}} \propto \int N_{\mathrm{e}}^{2} \mathrm{~d} R \sim\left(\mathcal{N}_{\mathrm{e}} / \pi R^{2} H\right)^{2} R$, where $\mathcal{N}_{\mathrm{e}}$ is the total electron number in the $\mathrm{CE}$ region studied, we can draw the following conclusion concerning the CE evolution as 

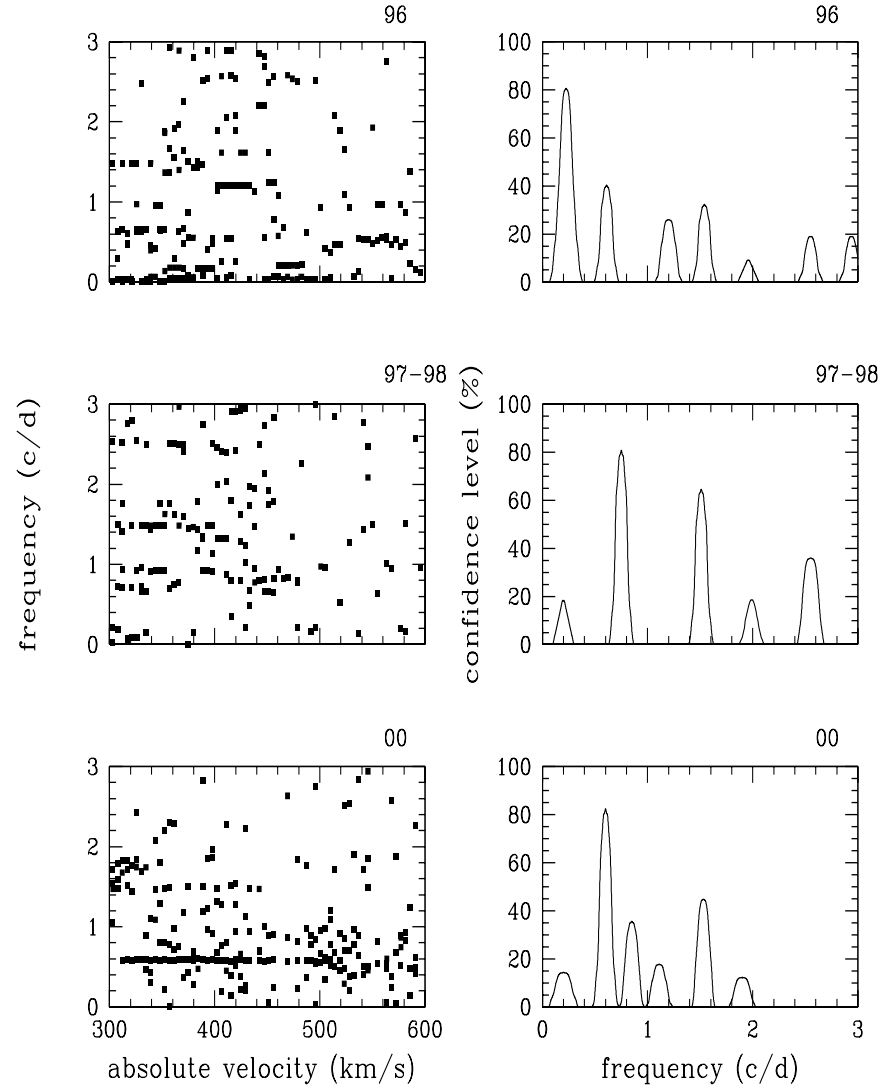

Fig. 15. Periodograms and confidence level diagrams for epochs 1996 , 1997/98 and 2000 of periodicities found in the wings of the $\mathrm{He}_{\mathrm{I}} 6678$ line beyond $v \sin i$. The red and blue sides of line profiles were folded into a unique positive velocity scale.

derived from the $\mathrm{H} \alpha$ line emission changes: from 1996 to 1997 these changes implied an increase of the emitting region by about $40 \%$ and an increase of $\mathcal{N}_{\mathrm{e}}$ by $60 \%$; the 1997-2000 transition is characterized by an increase of the emitting region extent by a factor 5.5 and $\mathcal{N}_{\mathrm{e}}$ by a factor 2.2; the 2000-2001 transition is almost passive as the emitting region shrank by about $60 \%$, while $\mathcal{N}_{\mathrm{e}}$ changed very little. The high NRP activity noticed in this work in the 2000 epoch may then correspond to an effective CE replenishing phase. Moreover, it is worth noticing that the nearly spectroscopic "shell" aspects of the $\mathrm{H} \alpha$ line in the 1996 and 1997 epochs that could be interpreted at first glance as due to an extended, highly absorbing disc, are actually produced by a $\mathrm{CE}$ region which accretes the ejected mass from the star in such a way that the region is rather close to the central star and that the extent of the region changes little.

\section{Conclusions}

In this paper we presented Fourier analysis of lpv of He I, Fe II, Mg II and Si II lines in the Be star $\eta$ Cen (HD 127972) observed in six epochs at high resolution and $S / N$ ratio from May 1996 to April 2001. The lpv were interpreted in terms of nonradial pulsations (NRPs). Time analysis was performed using Cleanest algorithm and showed the following frequencies with high order of significance: $0.61 \mathrm{c} / \mathrm{d}, 1.48 \mathrm{c} / \mathrm{d}, 3.81 \mathrm{c} / \mathrm{d}, 5.31 \mathrm{c} / \mathrm{d}$, $9.24 \mathrm{c} / \mathrm{d}$ and $10.35 \mathrm{c} / \mathrm{d}$. All signals except the first are

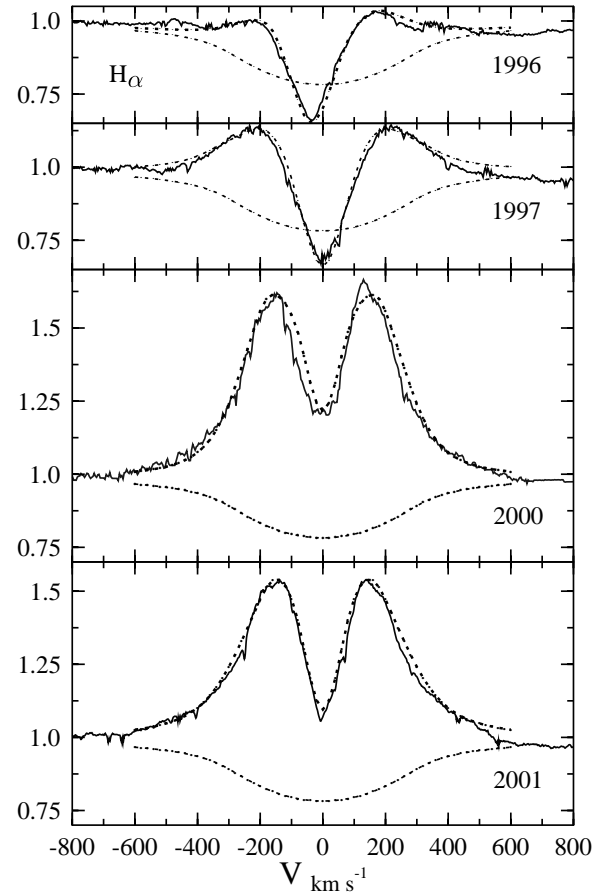

Fig. 16. Fits of observed $\mathrm{H} \alpha$ line emission profiles. The photospheric absorption component is also shown.

Table 6. CE parameters from fits of $\mathrm{H} \alpha$ emission line profiles.

\begin{tabular}{ccccccccc}
\hline \hline \multirow{2}{*}{ Epoch } & \multirow{2}{*}{$\tau_{\mathrm{o}}$} & \multirow{2}{*}{$R / R_{*}$} & $H / R_{*}$ & \multicolumn{2}{c}{$V_{\Omega} V_{\mathrm{r}}$} & $V_{\mathrm{P}}$ & \multirow{2}{*}{$\left(R / R_{*}\right)_{K}$} \\
\cline { 5 - 7 } & & & & \multicolumn{4}{c}{$\mathrm{km} \mathrm{s}^{-1}$} \\
\hline 1996 & 0.9 & 3.5 & 3.1 & 260 & 40 & 210 & 2.2 \\
1997 & 1.5 & 3.9 & 3.6 & 280 & 0 & 230 & 1.8 \\
2000 & 0.7 & 7.3 & 5.5 & 260 & 0 & 150 & 4.3 \\
2001 & 1.3 & 6.3 & 4.7 & 260 & 0 & 140 & 4.9 \\
\hline
\end{tabular}

interpreted as due to NRP modes. From phase variation diagrams we estimated $\ell$ parameters in the range $3-7$. If the $10.35 \mathrm{c} / \mathrm{d}$ frequency is considered the first harmonic of $5.31 \mathrm{c} / \mathrm{d}$, the corresponding azimuthal number of the mode is $|m|=4 \pm 2$. The observed multiperiodicity is in agreement with our latest works (Janot-Pacheco et al. 1999). The detected signal with $1.48 \mathrm{c} / \mathrm{d}$ is probably due to an NRP mode rather than to rotation, which is $v_{\mathrm{r}} \simeq 1.12$ to $1.24 \mathrm{c} / \mathrm{d}$ (at most $v_{\mathrm{r}}=1.39 \mathrm{c} / \mathrm{d}$ if the star were to be regarded as a critical rotator). The $3.81 \mathrm{c} / \mathrm{d}$ frequency could be interpreted as the multiple of $1.8 \mathrm{c} / \mathrm{d}(3.8 \sim$ $2 \times 1.9$ ), but $1.8 \mathrm{c} / \mathrm{d}$ was not found in LNA spectra (He I $6678 \AA$ ). It was detected in other wavelengths of ESO spectra. If this signal really existed, $3.81 \mathrm{c} / \mathrm{d}$ could be also interpreted as due to NRP. The only instance where we detected at the same time both a signal and its first harmonic was in the case of $5.31 \mathrm{c} / \mathrm{d}$ and $10.35 \mathrm{c} / \mathrm{d}$ signals, though the significance level of the latter frequency is less than $75 \%$. In this case, the IPS method yields a tesseral mode $\ell=5,|m|=4$.

The photometric variations of HD 127972 observed by Hipparcos from 1990 to 1992 were also considered. They could be folded with a frequency $v_{\text {phot }}=1.55 \mathrm{c} / \mathrm{d}$. According to the fundamental stellar parameters, this signal cannot be attributed to stellar rotation. 
The fundamental stellar parameters were determined by taking into account the first order effects produced by fast stellar rotation on the $\mathrm{BCD}$ spectrophotometric quantities used to estimate them. According to these parameters, the star should be in the midst of its MS life span as expected by Fabregat \& Torrejon (2000) for Be stars in open clusters. This also implies that, if the star becomes a fast rotator due to evolutionary effects as predicted by Maeder \& Meynet (2001), the object would need to be a rapid rotator on the ZAMS.

The $\mathrm{H} \alpha$ line profile was observed from May 1996 to April 2001. Its emission increased from 1996 to 2000 and then decreased slightly in 2001. The season-averaged $\mathrm{H} \alpha$ line emission strengthening was correlated with an increase of the lpv wavelength extent in the He I $\lambda 6678$ line (Figs. 11 and 14). The activity with high confidence level extended beyond the $V \sin i$ limit by $26 \%$ in 1997 and $45 \%$ in 2000 . The signal $v=0.6 \pm 0.1$ in the $\mathrm{He}_{\mathrm{I}} \lambda 6678$ line wings beyond the $V \sin i$ limit has an increasing level of significance from 1996 to 2000, which may indicate that the amount of matter gathered in the CE regions near the central star was growing during this period.

We used a simple model to study the characteristics of the season-averaged $\mathrm{H} \alpha$ line emission profiles. The fit of model emission line profiles indicates that the $\mathrm{H} \alpha$ variation from 1996 to 2000 is consistent with an increase of the amount of accumulated matter in the emitting region and with an enlargement of its extent. The 2000 to 2001 transition phase can be interpreted as due to shrinkage of the $\mathrm{CE}$ emitting region, where the amount of stored mass remained nearly unchanged.

Acknowledgements. RSL expresses his thanks to Dr. S. Andrievsky for fruitful discussions and help with line profile synthesis. He also thanks Dr. I. Hubený and Dr. T. Lanz for assistance with SYNSPEC and TLUSTY codes. We greatly appreciate and are greatful for the valuable comments and suggestions made by an anonymous referee. This research was supported by Fundação de Amparo à Pesquisa do Estado de São Paulo through grants no. 98/00497-0 and 00/10029-6 and Conselho Nacional de Desenvolvimento Científico e Tecnológico through grant no. 130710/1998-9.

\section{References}

Ando, H. 1983, A\&A, 35, 343

Ando, H. 1986, A\&A, 163, 97

Andrillat, Y., Jaschek, M., \& Jaschek, C. 1986, A\&AS, 65, 1

Baade, D. 1990, in ed. L. A. Willson, \& R. Stalio, Angular Momentum and Mass Loss for Hot Stars, NATO ASI Series C, 316, 177

Baillet, A., Chalonge, D., \& Divan, L. 1973, Nouv. Rev. Opt., 4, 3, 151

Balona, L. A. 1990, MNRAS, 245, 92

Balona, L. A. 1995, MNRAS, 277, 1547

Balona, L. A. 1999, MNRAS, 306, 407

Bjorkman, J. E., \& Cassinelli, J. P. 1993, ApJ, 409, 429

Chalonge, D., \& Divan, L. 1952, Ann. Astrophys., 15, 201

Chauville, J., Zorec, J., \& Ballereau, D., et al. 2001, A\&A, 378, 861

Cuypers, J., Balona, L. A., \& Marang, F. 1989, A\&AS, 81, 15

Collins II, G. W., \& Sonneborn, G. H. 1977, ApJS, 34, 41

Collins II, G. W., Truax, R. J., \& Cranmer, S. R. 1991, ApJS, 77, 541

Divan, L., \& Zorec, J. 1982, ESA-SP, 117, 101

Dobrichev, V. 1984, Comptes Rendus de l'Académie Bulgare des Sciences, 37(8), 991
Emilio, M., MSc Thesis 1997, IAGUSP

Endal, A. S., \& Sofia, S. 1979, ApJ, 232, 531

ESA 1997, The Hipparcos and Tycho Catalogues, ESA SP-120

Fabregat, J., \& Torrejon, J. M. 2000, A\&A, 357, 451

Feinstein, A., \& Marraco, H. G. 1979, AJ, 84, 1713

Floquet, M., Hubert, A. M., Hirata, R., et al. 2000, A\&A, 362, 1020

Floquet, M., Neiner, C., Janot-Pacheco, E., et al. 2002, A\&A, 394, 137

Foster, G. 1995, AJ, 109, 1989

Frémat, Y., \& Zorec, J. 2002, in Modelling of Stellar Atmospheres, IAU Symp. No 210, ed. N. E. Piskunov, W. W. Weiss, \& D. F. Grey (in press)

Frémat, Y., Zorec, J., Hubert, A. M., et al. 2002, A\&A, 385, 986

Frost, S. A., \& Conti, P. S. 1976, in Be and Shell Stars, ed. A. Slettebak (Reidel Publ. Co.), IAU Symp, 70, 139

Hanuschik, R. W., Hummel, W., Sutorius, E., et al. 1996, A\&AS, 116, 309

Hanuschik, R. W., Dachs, J., \& Baudzus, M., et al. 1993, A\&A, 274, 356

Harmanec, P. 2000, in The Be Phenomenon in Early-Type Stars, IAU Coll. No 175, ed. M. A. Smith, H. F. Henrichs, \& J., Fabregat, ASP Conf. Ser., 214, 13

Hauck, B., \& Mermilliod, M. 1998, A\&AS, 129, 431

Hubeny, I. 1988, Comp. Phys. Comm., 52, 103

Hubeny, I., Hummer, D. G., \& Lanz, T. 1994, A\&A, 282, 151

Hubert, A. M., Floquet, M., \& Zorec, J. 2000, in The Be Phenomenon in Early-Type Stars, IAU Coll. No 175, ed. M. A. Smith, H. F. Henrichs, \& J. Fabregat, ASP Conf. Ser., 214, 348

Hummel, W. 1994, A\&A, 289, 458

Janot-Pacheco, E., Leister, N. V., Hubert, A. M., Floquet, M., \& Hubert, H. 1991, Information Bulletin on Variable Stars, 3651, 1

Janot-Pacheco, E., Jankov, S., Leister, N. V., et al. 1999, A\&AS, 137, 407

Jaschek, C., Jaschek, M., \& Kucewicz, B. 1964, ZAp, 59, 108

Jefferies, J. T. 1968, Spectral Line Formation (Blaisdell Publishing Company, Massachusetts)

Johnson, H. L., \& Mitchell, R. I. 1975, Rev. Mex. Astron. Astrofis., 1, 299

Kambe, E., Ando, H., \& Hirata, R. 1993, A\&A, 273, 435

Kurucz, R. L. 1994, SAO CDROM N19

Lamers, H. J. G. L. M., \& Pauldrach, A. W. A. 1991, A\&A, 244, L5

Leister, N. V., Janot-Pacheco, E., Hubert, A. M., et al. 1994, A\&A, 287,789

Levenhagen, R. S., MSc Thesis 2000, IAGUSP

Maeder, A., \& Meynet, G. 2001, A\&A, 361, 101

Maeder, A., \& Peytremann, E. 1970, A\&A, 7, 120

Maeder, A., \& Peytremann, E. 1972, A\&A, 21, 279

Marlborough, J. M. 2000, in IAU Coll. 175, ed. M. A. Smith, H. F. Henrichs, \& J. Fabregat, ASP Conf. Ser., 214, 272

Moon, T. T., \& Dworetsky, M. M. 1985, MNRAS, 217, 305

Meynet, G., \& Maeder, A. 2000, A\&A, 361, 101

Mihalas, D. 1978, Stellar Atmospheres (San Francisco: W. H. Freeman, \& Co.)

Moss, D., \& Smith, R. C. 1981, Rep. Prog. Phys., 44, 831

Napiwotzki, R., Schönberner, D., \& Wenske, V. 1993, A\&A, 268, 653

Osaki, Y. 1986, PASP, 98, 30

Piskunov, N. E. 1992, in Stellar Magnetism, Proc. of International Meeting on Physics and Evolution of Stars, ed. Y. V. Glagolevskij, \& I. I. Romanyuk (Saint Petersburg: Nauka), 92

Rivinius, Th., Štefl, S., \& Baade, D. 1999, A\&A, 348, 831

Roberts, D. H., Lehar, J., \& Dreher, J. W. 1987, AJ 93, 968

Saio, H. 1994, in Pulsation, Rotation and Mass Loss in Early-Type Stars, ed, L. A. Balona, H. F. Henrichs, \& J. M. Le Contel (Kluwer), IAU Symp., 162, 287 
Schaller, G., Schaerer, D., Meynet, G., et al. 1992, A\&AS, 96, 269

Secchi, A. 1867, Astron. Nachrichten, 68, 63

Slettebak, A., Collins II, G. W., Boyce, P. B., et al. 1975, ApJS, 29, 137

Slettebak, A. 1982, ApJS, 50, 55

Smith, M. A. 1977, ApJ, 215, 574

Smith, M. A., \& Polidan R. S. 1993, ApJ, 408, 323

Snow, T. P. 1987, in Physics of Be Stars, ed. A. Slettebak, \& T. P. Snow (Cambridge Univ. Press), IAU Coll, 92, 250

Štefl, S., Baade, D., Harmanec, P., et al. 1995, A\&A, 294, 135

Schrijvers, C., \& Telting, J. H. 1999, A\&A, 342, 453

Struve, O. 1931, ApJ, 73, 94

Tassoul, J. L. 1978, Theory of Rotating Stars (Princeton University Press, Princeton, New Jersey)

Telting, J. H., \& Schrijvers, C. 1997a, A\&A, 121, 343

Telting, J. H., \& Schrijvers, C. 1997b, A\&A, 317, 742

Thomas, R. N. 1965, Nno-Equilibrium Thermodynamics in the Presence of a Radiation Field (Univ. Colorado Press, Boulder, Colorado)

Walker, G. A. H. 1991, in Rapid Variability of OB-Stars: Nature and Diagnostic Value, ESO Conference and Workshop Proceedings No. 36, ed. D. Baade (ESO), 27
Wolf, R. E. A. 1973, A\&A, 26, 127

Zahn, J. P. 1983, in Astrophysical Processes in Upper Main Sequence Stars, 13th Advanced Course of Saas Fee, Observatoire de Genève, 253

Zorec, J., \& Briot, D. 1991, A\&A, 245, 150

Zorec, J., \& Briot, D. 1997, A\&A, 318, 443

Zorec, J., Frémat, Y., \& Hubert, A. M. 2000a, in The Be Phenomenon in Early-Yype Stars, IAU Coll 175, ed. M. A. Smith, H. F. Henrichs, \& J. Fabregat, ASP Conf. Ser., 214, 330

Zorec, J., Frémat, Y., Hubert, A. M., \& Floquet, M. 2002a, in Radial and Nonradial Pulsations as probes of Stellar Physics, IAU Coll. No. 185, ed. C. Aerts, T. R. Bedding, \& J. Christensen-Dalsgaard, ASP Conf. Ser., 259, 244

Zorec, J., Frémat, Y., Hubert, A. M., et al. 2002b, in Modeling of Stellar Atmospheres, IAU Symp. 210, ed. N. E. Piskunov, W. W. Weiss, \& D. F. Gray, in press

Zorec, J., Hubert, A. M., \& Moujtahid, A. 2000b, in The Be Phenomenon in Early-Type Stars, IAU Coll 175, ed. M. A. Smith, H. F. Henrichs, \& J. Fabregat, ASP Conf. Ser., 214, 448

Zorec, J., Mochkovitch, R., \& Divan, L. 1988, C. R. Acad. Sci. Paris, 306, 1265 